\title{
A Complete Method for Assessing the Effectiveness of Eyewitness Identification
} Procedures: Expected Information Gain

\author{
Jeffrey J. Starns, Andrew L. Cohen, and Caren M. Rotello \\ Department of Psychological and Brain Sciences \\ University of Massachusetts, Amherst
}

\section{Author Note:}

Preparation of this article was supported by Grant No. 1454868 from the National Science Foundation.

Correspondence concerning this article should be addressed to Jeffrey Starns, Department of Psychological and Brain Sciences, University of Massachusetts, Amherst, Tobin Hall, 135 Hicks Way, Amherst, MA 01003, United States. Email: jstarns@umass.edu. 


\begin{abstract}
We present a method for measuring the efficacy of eyewitness identification procedures by applying fundamental principles of information theory. The resulting measure evaluates the Expected Information Gain (EIG) for an identification attempt, a single value that summarizes an identification procedure's overall potential for reducing uncertainty about guilt or innocence across all possible witness responses. In a series of theoretical demonstrations, we show that EIG often disagrees with existing measures (e.g., diagnosticity ratios or area under the ROC) about the relative effectiveness of different identification procedures. Each demonstration is designed to highlight "blind spots" of the existing measures as a contrast to EIG, which considers every factor relevant to a procedure's potential for decreasing uncertainty about guilt or innocence. Collectively, these demonstrations show that EIG has substantial potential to inspire new discoveries in eyewitness research. For research designed to identify procedures that will be most effective in criminal investigations, EIG supersedes all other measures, on both theoretical and practical grounds.
\end{abstract}

Keywords: Eyewitness identification, lineup, show-up, Shannon information, information theory 


\section{A Complete Method for Assessing the Effectiveness of Eyewitness Identification Procedures: Expected Information Gain}

Many critical functions of the criminal justice system hinge on the ability to perform a fundamental decision task; namely, judging which suspects are guilty and which are innocent. A perfect system would be capable of determining a suspect's guilt or innocence with $100 \%$ accuracy. In reality, of course, criminal justice officials must cope with many sources of uncertainty and make the best judgments possible given the available evidence. This challenge applies in obvious cases, such as a jury deciding whether or not to convict a defendant, but it is also manifest at many other decision points, such as a judge deciding whether the current evidence against a suspect justifies issuing a search warrant or a detective deciding whether the evidence against one suspect is sufficient to terminate investigation of other suspects. In scenarios like these, decision makers can achieve better outcomes if they have access to better evidence, that is, evidence that supports a higher level of certainty about the guilt or innocence of the suspect. Thus, developing principled methods for assessing the effectiveness of an evidencegathering procedure is a critical goal for effective research and practice. This goal goes beyond the question of how to make optimal use of evidence once it becomes available and necessitates a measure that can be used to compare different policies for obtaining new evidence.

One well-studied source of evidence in criminal investigations is an eyewitness identification of the suspect (Wells \& Olson, 2003). Identification attempts come in a wide variety of formats (National Research Council, 2015; Police Executive Research Forum, 2013). In the simplest form, the suspect is presented to the witness - either in person or in a picture and the witness indicates whether or not the suspect is the person they saw commit the crime. This procedure is known as a show-up, a name inspired by the practice of apprehending a suspect 
soon after a crime and "showing up" at the crime scene to present the suspect to a witness who is still being interviewed. Another common procedure is to present a suspect along with some number of fillers who are known to be innocent, creating a lineup. For the show-up procedure, a witness can either affirm that the suspect is the culprit (an identification) or fail to do so (a rejection). Lineups have an additional possible outcome, as the witness might claim that one of the fillers was the culprit (a filler identification). These basic witness responses are sometimes supplemented with ancillary information, such as the witness' level of confidence in their choice (Dallenbach, 1913; Deffenbacher, 1980) or the response time (RT) associated with the decision (Smith, Lindsay, \& Pryke, 2000; Sporer, 1992).

The ultimate goal of an identification attempt is to use the witness response to judge whether or not the suspect is guilty (Clark, 2012; Wells \& Lindsay, 1980; Wells, Yang, \& Smalarz, 2015). Decades of eyewitness memory research demonstrates that the success of this enterprise varies widely across situations; for example, positive identifications isolate guilty suspects with extreme reliability in some circumstances (Seale-Carlisle, Wetmore, Flowe, \& Mickes, 2019), but are very likely to promote false convictions of innocent suspects in other circumstances (Gronlund, Carlson, Dailey, \& Goodsell, 2009). The factors affecting the effectiveness of identification procedures include system variables that are under the control of the identification administrator and estimator variables that cannot be controlled but could potentially be assessed to aid in the interpretation of witness responses (Fulero, 2009; Wells \& Olson, 2003). Eyewitness researchers have explored a wide range of system variables such as whether the suspect is presented to the witness alone (i.e., in a show-up) or as a member of a lineup (e.g., Wetmore et al., 2015), whether lineup members are presented simultaneously or sequentially (e.g., Mickes, Flowe, \& Wixted, 2012), whether the witness is encouraged to make 
an identification (e.g., Steblay, 1997), and many others. Researchers have also thoroughly tested many estimator variables such as the viewing conditions of the witnessed crime (e.g., Lindsay, Semmler, Weber, Brewer, \& Lindsay, 2008; Loftus, 1985; Shapiro \& Penrod, 1986), whether the race of the witness is the same as that of the suspect (Malpass \& Kravitz, 1969), and the level of confidence reported by a witness (Dallenbach, 1913). We will define an identification procedure in terms of both system and estimator variables; for example, different procedures might be distinguished by the number of fillers in a lineup (system variable) or by the length of time that the culprit's face was in view of the witness (estimator variable).

Given the huge range of effectiveness across different identification procedures, researchers need to be able to assess a given procedure's potential to inform judgments about guilt or innocence. Researchers have compared the effectiveness of identification procedures using a range of quantitative measures such as the change in the probability of guilt from before to after an identification (Wells \& Lindsay, 1980), diagnosticity ratios (Clark, 2012), area under the receiver operating characteristic (ROC; Mickes et al., 2012), deviation from perfect performance (Smith, Lampinen, Wells, Smalarz, \& Mackovichova, 2019), and expected utility (Ceci \& Freidman, 2000). The relative merits of these measures are a matter of continuing debate (Lampinen, 2016; Lampinen, Smith, \& Wells, 2019; Rotello \& Chen, 2016; Smith, Wells, Smalarz, \& Lampinen, 2018; Wixted \& Mickes, 2012; Wixted, Mickes, Wetmore, Gronlund, \& Neuschatz, 2017). This issue is not merely academic; eyewitness research influences policies adopted by individual investigative units or recommended by government agencies (National Institute of Justice, 1999; National Research Council, 2014), and sometimes different measures support different policy recommendations (e.g., Wixted \& Mickes, 2012). Thus, identifying a comprehensive measure of the evidentiary value of an identification procedure is critical for 
driving future progress in eyewitness research and ensuring that investigators are both obtaining the best possible information from witnesses and interpreting that information in the most useful manner.

The approach we outline here is closely related to past work using Bayesian inference to evaluate identification procedures (Wells \& Lindsay, 1980; Wells \& Olson, 2002; Wells \& Turtle, 1986; Wells, Yang, \& Smalarz, 2015). The key insight of these studies is that the evidentiary value of a witness response can be measured by contrasting the prior probability of guilt (before the identification attempt) with the posterior probability of guilt (after the attempt). Bayes' theorem defines the shift from prior to posterior based on the likelihood of obtaining the observed witness response for guilty suspects and for innocent suspects (Wells \& Lindsay, 1980). We will build on this key innovation by measuring the Expected Information Gain (EIG) from the prior to the posterior distribution of uncertainty about guilt. ${ }^{1}$ EIG is calculated by applying information theory principles to eyewitness identification data; as such, it adopts a similar approach to some forensic applications in engineering (Ramos \& Gonzalez-Rodriguez, 2008; Ramos, Gonzalez-Rodriguez, Zadora, Zieba-Palus, \& Aitken, 2007).

Using EIG achieves two key advances over past applications of the Bayesian approach. Past studies have separately analyzed individual outcomes, such as a witness identifying the suspect (e.g., Wells \& Lindsay, 1980; Wells \& Olson, 2002; Wells \& Turtle, 1986; Wells, Yang, \& Smalarz, 2015). The first key advance is that EIG measures the overall evidentiary potential of a procedure across a number of identification attempts with varying outcomes. Thus, EIG provides a single index of the overall efficacy of an identification procedure. This advance

\footnotetext{
${ }^{1}$ The expected information gain for random variable A produced by learning the outcome of random variable B is also commonly called the mutual information of A and B (Cover \& Thomas, 2007).
} 
allows researchers to select the optimal procedure in the many cases with tradeoffs in evidentiary value (e.g., a procedural change makes identifications stronger evidence of guilt but also renders rejections weaker evidence of innocence; Clark, 2012; Wells, Yang, \& Smalarz, 2015). It also allows researchers to compare procedures with non-corresponding response options, such as procedures with and without confidence ratings. Finally, it gives researchers a way to evaluate predictors of witness accuracy, such as the response time associated with the identification decision (e.g. Sporer, 1992), by contrasting the evidentiary value of witness responses with and without consideration of the predictor variable.

Past studies have typically used "raw" probability to index the information provided by identification procedures. For example, if the probability of guilt was $50 \%$ before a witness identification attempt and $80 \%$ after, this change was described as an information gain of $30 \%$ (Wells \& Lindsay, 1980). The second key advance is that EIG uses Shannon surprisal (Shannon, 1948) to measure the degree of error in probabilistic judgments, which confers a number of key advantages over measures on a probability scale (Gneiting \& Raftery, 2007; McElreath, 2016). Indeed, Shannon surprisal has proven to be an invaluable tool in computer science (Chaitin, 1975), medicine (Benish, 1999), and engineering (Cover \& Thomas, 2006). Techniques based on Shannon surprisal are beginning to play a critical role in forensic applications (Ramos et al., 2007), such as developing automated recognition systems for faces (Gong, Li, Tao, Liu, \& Li, 2015), speakers (Campbell, Reynolds, Campbell, \& Brady, 2005), or fingerprints (Osterburg, Parthasarathy, Raghavan, \& Sclove, 1977).

Investigators cannot reasonably adopt unique identification procedures for each individual suspect, so eyewitness research should focus on identifying procedures that are effective when applied across a large set identification attempts. Shannon surprisal possesses a 
critical property that supports this goal: It measures uncertainty on an additive scale (Shannon, 1948). That is, summing (or averaging) surprisal values accurately characterizes the total (or average) uncertainty for a set of judgments. This property will play a key role in many of the following illustrations of the advantages of EIG. For example, being able to average uncertainty across outcomes supports the first key advance by allowing researchers to discover which identification procedures are the most effective when used across many different identification attempts even though the witness responses will vary from one attempt to the next.

Together, these two developments make EIG an extremely useful research tool that is theoretically grounded in a well-developed and highly influential method of quantifying information (Shannon, 1948).

\section{Overview}

In what follows, we (1) explain the principles that motivate the EIG measure, (2) provide equations for calculating EIG from eyewitness identification data, and (3) present a series of theoretical demonstrations designed to help readers understand the properties of EIG. Our demonstrations focus on exploring differences between EIG and existing measures, and we will establish that EIG has the potential to both redefine interpretations of existing results and inspire important new directions in eyewitness memory research.

The remainder of the paper is organized as follows. The Information Theory and Identifications section explains the basis for the EIG measure, which combines a Bayesian approach to interpreting witness responses (Wells \& Lindsay, 1980) with foundational measures from information theory (Shannon, 1948). Using a simple hypothetical data set, we (1) review the process of using Bayes' rule to guide probabilistic judgments of guilt or innocence; (2) explain how Shannon surprisal provides a measure of the degree of error in these probabilistic 
judgments; (3) demonstrate that Shannon surprisal provides a scoring rule for judgment error that is fully consistent with Bayes' theorem and permits valid averaging across multiple judgments by putting uncertainty on an additive scale; (4) introduce the concept of entropy, the average judgment error across a probability distribution, and explain how it quantifies uncertainty about guilt or innocence; and (5) show how the change in entropy produced by witness responses can be used to calculate EIG. In short, this section presents the full logic that supports EIG as a measure of evidentiary value.

The EIG and EPG Equations for Eyewitness Researchers section provides a complete set of equations for calculating EIG that can be used across a variety of identification experiments regardless of the specific format (e.g., show-up or lineup) and response options (e.g., lineups that do or do not include confidence ratings). This section also provides equations for translating EIG back to the more familiar probability scale for ease of interpretation, yielding a value we call Expected Probability Gain (EPG).

The Demonstrations Exploring EIG section presents a series of example applications designed to illustrate key properties of EIG. A common theme throughout the demonstrations is that EIG considers all factors relevant to the evidentiary value of an identification procedure, and we highlight situations in which EIG's comprehensive assessment supports conclusions that differ from existing measures. We also provide examples of how EIG can inspire new lines of inquiry in eyewitness memory research. We use well-validated decision models to generate example data sets for the demonstrations, which allows us to ensure that we are working with realistic data patterns while also isolating the key processes that distinguish EIG from existing measures. Thus, we establish that EIG is an important measure on purely theoretical grounds, and provide a roadmap for future empirical conclusions. 
We report five demonstrations in total. The first demonstration contrasts EIG and diagnosticity ratios. Diagnosticity ratios for witness identifications indicate higher evidentiary value when witnesses are more conservative; that is, when they are reluctant to make an identification unless they are very confident that the suspect is the culpri. In contrast, EIG reveals that any bias in responding, whether liberal or conservative, limits the evidentiary value of witness responses. This demonstration also reveals that EIG is affected by the prior probability of guilt, but that researchers can identify useful identification procedures even when the prior probability of guilt varies widely across different identification attempts, as it would in real investigations. Indeed, we show that optimizing EIG under the assumption of a 50\% prior probability of guilt identifies near-optimal procedures for any actual prior probability.

The second demonstration contrasts EIG with area under the Receiver Operating Characteristic (ROC; Macmillan \& Creelman, 2005; Mickes et al., 2012). The key conclusion from this section is that these two measures are designed to measure subtly different constructs: Whereas area under the ROC measures how well witnesses can discriminate guilty and innocent suspects based on their memory for the culprit, EIG measures how well the witness responses can be used by an outside observer - whether it be a detective, juror, or judge - to improve assessments of a suspect's guilt or innocence. (Wells, Yang, \& Smalarz, 2015, also noted this distinction in comparing the ROC and Bayesian approaches). The former construct is often very useful for clarifying theoretical issues, but the latter construct is the proper focus when the goal is to use data from eyewitness memory experiments to support policy recommendations about procedures adopted in real investigations. We also present an example evaluating variation in lineup size which shows that EIG and area under the ROC lead to different conclusions in some situations. 
The third demonstration contrasts EIG and Deviation from Perfect Performance (DPP; Smith et al., 2019) by exploring the potential evidentiary value of obtaining confidence ratings from witnesses. For example, witnesses might rate their confidence that the suspect is the culprit on a scale of 0-100 (e.g., Colloff \& Wixted, 2020). The DPP approach collapses the confidence rating scale at different cutoff values to find the one that produces the lowest overall error rate, with all confidence ratings below the cutoff value collapsed into the "rejection" category and all confidence ratings above the cutoff value collapsed into the "identification" category. EIG reveals that collapsing a confidence scale in this way substantially reduces the evidentiary value of witness responses compared to considering all confidence levels separately. Thus, DPP and similar measures cannot capture the full evidentiary potential of an identification procedure.

The fourth demonstration contrasts EIG and expected utility. We show that these two approaches are fundamentally very similar; indeed, the only distinction between the two is that expected utility requires researchers to specify the instrumental value of identification outcomes whereas EIG evaluates the intrinsic evidentiary value of these outcomes. We note that instrumental values are nearly always unknown and vary widely across different types of decisions that might be informed by an identification procedure, so eyewitness researchers should focus on finding procedures that optimize evidentiary value.

The fifth, and final, demonstration shows how EIG can be used to evaluate continuous predictors of witness accuracy, such as the response time (RT) of identification responses. EIG reveals that having access to variables that predict witness accuracy increases the evidentiary value of witness responses. This example outlines a potential research application of EIG that cannot be supported by any of the existing measures. 
We have included a number of Conceptual Summary statements, typically at the end of major sections, that review important concepts without quantitative detail. Readers who are not interested in mathematical implementation can skip from the section introductions to the conceptual summaries to obtain a complete picture of our major claims and the qualitative arguments that support them.

\section{Information Theory and Identifications}

This section introduces the mathematics for analyzing eyewitness identifications that are applied in the demonstrations reported below. Our goal is to have a single measure representing the overall potential of a given identification procedure to provide information about guilt or innocence, which, in turn, will allow researchers to directly compare different procedures and inform policy recommendations for investigative practices.

The critical steps in pursuing this goal are (1) use Bayes' theorem to assess the probability that a given suspect is guilty given the identification response (Wells \& Lindsay, 1980), (2) use Shannon surprisal to measure the degree of error in probabilistic judgments (Shannon, 1948), (3) evaluate the average judgment error, or entropy, across a full probability distribution representing uncertainty about suspects' guilt or innocence, (4) calculate the change in entropy, or information gain, from the prior distribution to the posterior distribution associated with each witness response; and (5) combine across all responses to yield an Expected Information Gain (EIG) score that characterizes the evidentiary value of the identification procedure as a whole. Within this framework, a procedure with high evidentiary value is one that tends to dramatically reduce uncertainty about guilt or innocence.

This section also discusses some of the important measurement considerations involved in selecting EIG as an index of overall evidentiary value. We demonstrate that EIG uses the same 
standard of success implied by the Bayesian approach; thus, it is a natural extension of previous work on Bayesian inference in identification tasks (Wells \& Lindsay, 1980; Wells \& Olson, 2002; Wells \& Turtle, 1986; Wells, Yang, \& Smalarz, 2015). We also explain why defining overall evidentiary value requires a measure that puts uncertainty on an additive scale, one of the key properties that has made Shannon surprisal an extremely useful measure of uncertainty (Cover \& Thomas, 2006; McElreath, 2016; Shannon, 1948; Quinlan, 1986). Finally, we introduce Expected Probability Gain (EPG), which may aid the interpretation of evidentiary value metrics by translating EIG to the probability scale.

Readers who are not interested in quantitative details can skip to the Conceptual Summary of Information Theory and Identifications sub-section at the conclusion of this section.

\section{Bayesian Updating After a Witness Response}

We will begin with a simple example illustrating how Bayes' theorem can be used to update the probability that a suspect is guilty based on a witness response, an important first step in assessing how an identification procedure reduces uncertainty about guilt or innocence (Wells \& Lindsay, 1980; Wells \& Olson, 2002; Wells \& Turtle, 1986; Wells, Yang, \& Smalarz, 2015). Our example takes the form of a hypothetical data set from an eyewitness memory experiment. This section shows how to calculate the prior and posterior probability of guilt associated with each possible witness response using Bayes' Rule. The following sections use these probabilities to compute EIG as a measure of evidentiary value.

Imagine an eyewitness identification experiment in which participants acting as witnesses (hereafter, "witnesses") viewed a simulated crime and then completed a show-up identification procedure, i.e., each witness saw a single suspect and decided whether or not the suspect was the culprit in the simulated crime (e.g., Gronlund, Carlson, Neuschatz, Goodsell, Wetmore, Wooten, 
$\&$ Graham, 2012). For simplicity, imagine that the only variable manipulated in the experiment was the amount of time that the culprit was in view: 10 seconds or 30 seconds (see Memon, Hope, \& Bull, 2003, for a similar manipulation). Our hypothetical experiment had 1000 witnesses in each of these viewing conditions, and half of the witnesses within each condition were tested with the guilty suspect (the culprit) and the other half were tested with an innocent suspect.

Table 1 displays a plausible pattern of results. For the 500 show-ups with the guilty suspect in the 10-second condition, $400(80 \%)$ resulted in identifications, i.e., the witness responded that the suspect was the culprit, leaving 100 (20\%) that resulted in rejections, i.e., the witness responded that the suspect was not the culprit. For the 500 show-ups with an innocent suspect in the 10-second condition, 50 (10\%) resulted in identifications, leaving 450 (90\%) rejections. Predictably, accuracy rates were higher in the 30-second viewing condition, with 450 (90\%) identifications and $50(10 \%)$ rejections for show-ups with the guilty suspect, and $25(5 \%)$ identifications and 475 (95\%) rejections for show-ups with an innocent suspect.

In the Bayesian approach to interpreting eyewitness identifications, uncertainty about the guilt or innocence of a particular suspect is represented by a probability distribution, and this distribution is updated when new evidence is encountered (Wells \& Lindsay, 1980). Here, there are two hypotheses that might be true (guilty or innocent), so uncertainty is represented by a Bernoulli distribution. The Bernoulli distribution is characterized by a single parameter, which, in this case, can be defined as the probability that the suspect is guilty, $p(g)$, where the probability that the suspect is innocent is the complement of this value, i.e., $p(i)=1-p(g)$. Because both conditions in the hypothetical data set included an equal number of show-up trials with a guilty suspect and an innocent suspect, initial uncertainty about guilt for a randomly 
sampled show-up trial is represented by a Bernoulli distribution with a $50 \%$ chance of guilt and a $50 \%$ chance of innocence. In other words, with no information about whether or not the witness identified the suspect, one is resigned to being completely uncertain about whether the suspect on a given trial is guilty or innocent.

Learning whether or not the suspect was identified by the witness would help to refine the possibilities, and Bayes' theorem defines how the arrival of new information changes the distribution of uncertainty over a set of hypotheses (Fienberg, 2006; McGrayne, 2011). For suspects who were identified, the updated probability of guilt is found by

$$
p(g \mid i d)=p(i d \mid g) p(g) /(p(i d \mid g) p(g)+p(i d \mid i) p(i))
$$

where $g$ represents a guilty suspect, $i$ represents an innocent suspect, and $i d$ represents a witness identification. The prior probabilities, $p(g)$ and $p(i)$, represent the probability of guilt and innocence before the witness identification is considered, respectively. Again, in our example, $p(g)=p(i)=0.50$. The terms $p(i d \mid g)$ and $p(i d \mid i)$ represent the likelihood of an identification when the suspect is guilty and innocent, respectively. In the 10 -second condition, $p(i d \mid g)=0.80$, because $80 \%$ of the trials with the guilty suspect produced an identification, and $p(i d \mid i)=0.10$, because $10 \%$ of the trials with an innocent suspect produced an identification. Given these values, Bayes' theorem can be used to compute the posterior probability, $p(g \mid i d)$. Plugging the current values into Equation 2, we have

$$
0.80 \times 0.50 /(0.80 \times 0.50+0.10 \times 0.50)=0.89
$$

meaning that the probability of guilt increases from $50 \%$ before an identification to $89 \%$ after an identification. In other words, the updated state of uncertainty is represented by a Bernoulli distribution with an $89 \%$ chance of guilt and, therefore, an $11 \%$ chance of innocence. For the 30-second condition, the updated (posterior) probability of guilt is given by, 


$$
0.90 \times 0.50 /(0.90 \times 0.50+0.05 \times 0.50)=0.95,
$$

so the updated state of uncertainty is represented by a Bernoulli distribution with a $95 \%$ chance of guilt and a 5\% chance of innocence. Comparing these conditions shows that identifications are a stronger indication of guilt in the 30 -second condition than in the 10 -second condition, which is a natural consequence of the fact that witnesses with a longer viewing opportunity did a better job of limiting their identifications to guilty suspects.

These same steps can also be used to determine how the probability of guilt changes after a rejection by the witness, $r e j$ :

$$
p(g \mid r e j)=p(r e j \mid g) p(g) /(p(r e j \mid g) p(g)+p(r e j \mid i) p(i)) .
$$

In the 10-second condition, this yields

$$
0.20 \times 0.50 /(0.20 \times 0.50+0.90 \times 0.50)=0.18,
$$

indicating that the probability of guilt decreases from $50 \%$ before a rejection to $18 \%$ after a rejection. In other words, the updated state of uncertainty is represented by a Bernoulli distribution with an $18 \%$ chance of guilt and an $82 \%$ chance of innocence. In the 30-second condition, the probability of guilt after a rejection is

$$
0.10 \times 0.50 /(0.10 \times 0.50+0.95 \times 0.50)=0.095 \text {, }
$$

and the posterior is a Bernoulli distribution with a $9.5 \%$ chance of guilt and a $90.5 \%$ chance of innocence. Thus, rejections are a stronger indication of innocence in the 30 -second than in the 10-second condition, a consequence of the fact that witnesses in the former condition did a better job of limiting their rejections to innocent suspects.

With the full table of results from the hypothetical experiment (Table 1), one can take a short cut to calculating the posterior probabilities. Taking the 10 -second condition as an example, out of 450 total identifications, witnesses made 400 identifications for show-ups with 
the guilty suspect, so the probability that the suspect is guilty for trials with an identification is $400 / 450=.89$, the same value calculated above. Similarly, out of 550 total rejections, witnesses made 100 rejections for show-ups with the guilty suspect, so the probability that the suspect is guilty for trials with a rejection is $100 / 550=.18$.

We presented a full application of Bayes' Theorem, rather than this shortcut, for two reasons. First, we will need to rely on the more general form for some of our equations. Second, the full application illustrates how the change from the prior to posterior probability distribution is determined by the likelihood of observing the evidence under each hypothesis (here, the likelihood of getting a particular witness response for guilty and innocent suspects).

This hypothetical data set would support the conclusion that increased viewing time enhanced the evidentiary value of the show-up procedure. Specifically, when the witness had the opportunity to view the culprit for a longer period of time, identifications were better evidence of guilt and rejections were better evidence of innocence. This conclusion is certainly consistent with the findings of real studies (e.g., Memon et al., 2003), and viewing conditions are classic estimator variables that should be considered when witness responses are used as sources of evidence about guilt or innocence (Shapiro \& Penrod, 1986). Eyewitness memory research plays a critical role in this process, and the Bayesian approach has provided valuable insight into effective policies for identification procedures (Wells \& Lindsay, 1980; Wells \& Olson, 2002; Wells \& Turtle, 1986; Wells, Yang, \& Smalarz, 2015). For example, Wells, Yang, and Smalarz (2015) explored a number of variables in terms of the shift from the prior to the posterior probability of guilt for individual witness responses.

We aim to extend the Bayesian approach with two key advances. First, we will measure the overall evidentiary value of an identification procedure by analyzing a full set of 
identification attempts simultaneously, rather than considering individual witness responses in isolation. A procedure adopted by real investigators will be used across numerous cases, so policy recommendations should prioritize procedures that are effective in general across all the possible witness responses. In our show-up example, viewing time was the only difference between conditions, and increased viewing time enhanced the evidentiary value of both identifications and rejections, so it was easy to identify the superior condition. Many potential differences in procedure, however, will enhance the evidentiary value of some witness responses and degrade the value of others (Clark, 2012); in fact, some of the variables investigated by Wells, Yang, and Smalarz (2015) showed exactly this pattern. Moreover, some comparisons involve procedures that do not have the same set of possible witness responses, such as procedures with and without confidence ratings. In these cases, determining which procedure has a higher overall evidentiary value requires averaging across all identification attempts even though the witness response changes from one attempt to the next.

Achieving the first advance necessitates a second. As detailed in the following section, we will substitute a measure of uncertainty that has advantageous measurement properties: Shannon surprisal (Shannon, 1948). Wells et al. (2015) used the term "information gain" to refer to a change in the probability of guilt, but we will adopt the more traditional meaning of information gain as a change in uncertainty measured on the Shannon surprisal scale (e.g., Quinlan, 1986). Research on scoring rules for probabilistic judgments has uncovered many desirable properties of measures that apply a logarithmic transformation to probabilities, like Shannon surprisal (e.g., Bickel, 2007; Savage, 1971; McElreath, 2016; Winkler, 1969). We will discuss a few critical advantages in a later section. Most importantly, Shannon surprisal puts uncertainty on an additive scale, so averaging scores on the surprisal scale accurately 
characterizes average uncertainty across multiple identification attempts (Shannon, 1948). The following section explains how Shannon surprisal measures the degree of error in probabilistic judgments, the next step in our pursuit of a comprehensive measure of evidentiary value.

\section{Measuring Judgment Error with Shannon Surprisal}

This section introduces the measurement scale used to define evidentiary value. In a general sense, the value of evidence is that it facilitates more accurate judgments about whether a suspect is guilty or innocent, so we seek a measure that quantifies this reduction in judgment error. Of course, the ultimate goal is to assign as much credibility as possible to the true state of affairs. If a suspect is innocent, for example, then judging that the suspect has an $98 \%$ chance of being innocent represents very low judgment error, judging that the suspect has a $75 \%$ chance of being innocent represents higher judgment error, and judging that the suspect has a 5\% chance of being innocent represents extremely high judgment error. Accordingly, the distributions of uncertainty about guilt discussed in the previous section (i.e., the probabilities associated with guilt and innocence) will now play the role of judgements of guilt. In the hypothetical show-up experiment, for instance, saying that a suspect who was rejected by the witness has an $18 \%$ chance of being guilty is equivalent to judging that a particular rejected suspect is innocent while acknowledging an $18 \%$ chance that the judgment is incorrect. Information theory provides an elegant and axiomatic way to measure the error of a probabilistic judgment, and we will now show how this foundational concept can form the basis of a comprehensive measure of the effectiveness of an identification procedure.

Although Shannon (1948) initially applied information theory to the communication of messages, it has proven important in a wide range of applications (Cover \& Thomas, 2005), including other areas of psychology (e.g., Attneave, 1959; Crupi, Nelson, Meder, Cevolani, \& 
Tentori, 2018) and forensics (e.g., Ramos \& Gonzalez-Rodriguez, 2008). The key idea is to define information as the reduction in uncertainty (or equivalently, the increase in certainty) after observing an outcome, where uncertainty is represented by a probability distribution over possible states of the world. Shannon (1948) derived the equation for the amount of information gained by a single outcome, or surprisal, to satisfy a number of reasonable and intuitive properties, among them: (1) a definite outcome produces no information, so zero on the scale represents the complete lack of uncertainty; (2) less probable outcomes produce more information; and (3) the information produced from multiple events is additive. There is a unique function that satisfies these axioms and has many other desirable properties. Namely, the surprisal or information content $I$ of an outcome $y$ is defined as

$$
I(y)=\log _{2}(1 / p(y)),
$$

where $p(y)$ is the judged probability of outcome $y$ before the outcome was revealed. The logarithm base is arbitrary, but, when base 2 is used, as we adopt here, information is measured in bits (Shannon, 1948). The left panel of Figure 1 shows how surprisal varies as a function of the judged probability of the outcome. Note that surprisal is higher for outcomes that were judged to be less probable, and that the function is steeper on the low end of the probability scale.

Surprisal is a natural measure for the degree of error in a probabilistic judgment of an unknown outcome, in this case, whether a suspect is guilty or innocent. For example, consider a suspect who is in fact guilty. A judgment representing high judgment error would assign a low probability of guilt, say $1 \%$. In this case, learning that the suspect is in fact guilty would be a big surprise, and, equivalently, a large gain in information:

$$
\log _{2}(1 / .01)=6.64 \text {. }
$$


A judgement representing low judgment error would assign a high probability of guilt, say $99 \%$. In this case, learning that the suspect is indeed guilty would not come as much of a surprise, and would therefore represent a small gain in information:

$$
\log _{2}(1 / .99)=0.01
$$

Conversely, if the suspect was in fact innocent, then the surprisal values would reverse to show that assigning a low probability of guilt (i.e., a high probability of innocence) represents a good judgment and assigning a high probability of guilt represents a bad judgment. For example, jurors who decided to convict a defendant because they were convinced of his guilt should be very surprised to learn that he was later exonerated by DNA evidence, and this surprisal signifies a high degree of error in their judgment (in this scenario, an error with disastrous consequences for the defendant).

Before discussing how to use Shannon surprisal to evaluate eyewitness identification procedures, we will briefly pause to consider whether the measure is well suited to the task. Extensive past work exploring different scoring rules for probabilistic judgments has uncovered some important advantages of using logarithmic measures like surprisal (e.g., Bickel, 2007). An extensive summary of this work is beyond the scope of this paper, but we will briefly note some particularly relevant considerations. We proceed by reevaluating parts of the previous hypothetical show-up experiment.

In the 10-second viewing condition, consider show-up trials with a suspect identification, $89 \%$ of which were trials with a guilty suspect. What is the best judgment about guilt to make for each individual identification attempt that produced a suspect identification? Intuitively, the appropriate option would be to judge that the suspect has an $89 \%$ chance of being guilty, or equivalently, to conclude that the suspect is guilty and acknowledge an $11 \%$ chance that this 
judgment could be incorrect. Indeed, if knowing that the witness identified the suspect is the only source of information about guilt, then one cannot achieve anything lower than an $11 \%$ error rate in judgements of guilt or innocence in this situation. As such, judging more than an $89 \%$ probability of guilt could be considered misguided at best, and dishonest at worst. It turns out that these intuitions are theoretically justified: Adopting a level of certainty appropriate for the actual error rate is a requirement of optimal decision making, as it allows the decision maker to anticipate the expected costs and benefits of different courses of action (Roberts, 2007). Thus, scoring-rule researchers have focused on identifying measures that are strictly proper, meaning that judgment error is uniquely minimized by probability judgments that match the true risk of making an error, or synonymously, judgments that are based on the appropriate application of Bayesian inference (e.g., Bickel, 2007; Gneiting \& Raftery, 2007; Savage, 1971; Winkler, 1969). According to a strictly proper scoring rule, good judgments are judgments that reflect an appropriate level of uncertainty and, thus, can support effective decision making.

The left panel of Figure 2 shows the average judgment error across the set of identified suspects from the 10-second viewing condition as indexed by Shannon surprisal. Considering only the guilty suspects, judgment error as measured by Shannon surprisal is minimized by reporting a $100 \%$ chance of guilt and increases at lower probabilities of guilt. Similarly, considering only the innocent suspects, judgment error is minimized by reporting a $0 \%$ chance of guilt (a 100\% chance of innocence) and increases for higher probabilities. Of course, identifications were not a perfect indication of guilt, so it is critical to evaluate the overall judgment error across the entire set of identifications, as shown by the solid purple line. This line shows the average of the functions for guilty and innocent suspects weighted by the proportion of trials with identifications that fell into each guilt category. Most trials that led to 
identifications had a guilty suspect, so the weighted average is closer to the guilty-suspect function. Critically, the nadir of the overall function lies at exactly $89 \%$. Thus, when Shannon surprisal is used to measure error, judgment error is minimized for the judgment that matches the probability of guilt determined by a Bayesian analysis of the evidence. Equivalently, this is the judgment that expresses a level of certainty that matches the true error rate supported by the available evidence.

The right panel of Figure 2 shows the same functions for show-up trials in which the witness rejected the suspect in the 10 -second viewing condition, $18 \%$ of which were trials with a guilty suspect. Most trials that led to rejections had an innocent suspect, so the weighted average is closer to the innocent-suspect function. Overall judgment error is minimized by judging an $18 \%$ chance of guilt after each rejection, or equivalently, judging that the suspect is innocent and being $82 \%$ certain that the judgment is correct.

The perfect balancing displayed in Figure 2 does not just occur when $89 \%$ or $18 \%$ of identification attempts involve a guilty suspect: it applies to every other probability of guilt supported by the available evidence (Winkler, 1969). Moreover, the perfect balancing is not a mere coincidence; it is a consequence of a basic mathematical principle. Uncertainty compounds multiplicatively on the probability scale; for example, if one needs to make two independent judgments with a $50 \%$ chance of getting each one correct, then the chance of getting both correct is $.5 \times .5=.25$. Using logarithmic measures transforms the multiplicative scale to an additive scale. For example, an outcome with $50 \%$ uncertainty has a Shannon surprisal of $\log _{2}(1 / .5)=1$. Similarly, two independent judgements with $50 \%$ uncertainty have a Shannon surprisal of $\log _{2}$ $(1 /(.5 \times .5))=2$, and an average surprisal of $2 / 2=1$. The additive scale correctly indexes the total or average uncertainty across a set of identification attempts when the individual scores are 
summed or averaged, and logarithmic measures are the only measures that put uncertainty on an additive scale (Shannon, 1948).

The Bayesian approach is widely advocated as the proper method for interpreting evidence (McGrayne, 2011), and we have just presented a justification for why we should evaluate evidence using Bayesian inference - it minimizes judgment error as measured by Shannon surprisal, a strictly proper measure that rewards judgments that support effective decision making. Although a few other scoring rules are also strictly proper, logarithmic-based measures like Shannon surprisal are the only ones that are fully consistent with Bayesian inference (Bickel, 2007; Winkler, 1969).

Taking stock, we have discussed the Bayesian method for assessing guilt based on identification outcomes, presented Shannon surprisal as a measure of the degree of error in probabilistic judgments, and noted the deep link between these two concepts. We now have an appropriate measurement scale to define the information gain associated with particular witness responses. The next section introduces the measure of uncertainty on the Shannon surprisal scale.

\section{Entropy and Uncertainty}

The average Shannon surprisal for a single probability distribution is known as entropy and is traditionally denoted with $H$ (Shannon, 1948). Let $Y$ be a discrete random variable with outcomes $y_{j}$, each with probability $p\left(y_{j}\right)$, then, the entropy of $Y$ is defined as

$$
H(Y)=\Sigma p\left(y_{j}\right) \log _{2}\left(1 / p\left(y_{j}\right)\right)
$$

That is, entropy is a sum over all possible outcomes in which the surprisal of each outcome is weighted by the probability of that outcome (i.e., the average surprisal). Because events that never happen cannot affect average surprisal, outcomes with probability zero are dropped. In 
short, entropy measures the uncertainty associated with a probability distribution - the less predictable the outcome, the higher the entropy.

In the current context, $Y$ has two possible outcomes: guilty, $g$, associated with probability $p(g)$, and innocent, $i$, associated with probability $p(i)$. Note that the surprisal values in the entropy formula are defined using the true probability of observing each outcome in the probability distribution. In the current context, this means that judgments of guilt or innocence are based on a proper interpretation of the evidence available. For example, if the evidence suggests that the suspect is guilty but is misleading $25 \%$ of the time, then judgments of guilt adopt a $75 \%$ level of certainty, i.e., the judged $p(g)=.75$ and the judged $p(i)=.25 .^{2}$ One nice consequence of this assumption is that the average judgment error is uniquely determined by the inherent uncertainty of a probability distribution, making entropy a pure measure of this uncertainty.

The right panel of Figure 1 shows how entropy for judgments about guilt or innocence changes across different distributions of uncertainty, with each distribution characterized by the probability that a suspect from the distribution is guilty, $p(g)$. Notice that entropy is highest at .5 and decreases as the probability of guilt moves closer to 0 or 1 . In everyday terms, entropy agrees with the intuition that it is easier to judge guilt for a set of predominantly guilty or predominantly innocent suspects than for a set that is an even mix of guilty and innocent suspects. If one must judge the guilt of suspects sampled from a set of $50 \%$ guilty and $50 \%$

\footnotetext{
${ }^{2}$ We acknowledge that human judgment is often misaligned with the evidence that supports it; for example, someone who has access to evidence that correctly indicates guilt or innocence $75 \%$ of the time might nonetheless be $95 \%$ certain in their judgments (Juslin, Olsson \& Winman 1996; Brewer, Keast, \& Rishworth, 2002). However, we are interested in measuring the potential value of evidence-gathering procedures, and thus we assume that the evidence that they provide is properly interpreted. It is trivial to note that any evidence-gathering procedure could be rendered irrelevant or even misleading if the evidence it provides is systematically misinterpreted. Of course, one of the primary functions of forensic research is facilitating appropriate interpretation of evidence-gathering procedures by investigating their reliability.
} 
innocent suspects, then the best one can do is judge that every suspect in the set has a 50-50 chance of being guilty or innocent. This leads to high judgment error, i.e., high surprisal at learning the true outcome, for both guilty suspects,

$$
I(g)=\log _{2}(1 / p(g))=\log _{2}(1 / 0.50)=1.00 \text { bit }
$$

and innocent suspects,

$$
I(i)=\log _{2}(1 / p(i))=\log _{2}(1 / 0.50)=1.00 \text { bit }
$$

and a high average judgment error (entropy) across the entire set of suspects,

$$
H(Y)=p(g) I(g)+p(i) I(i)=0.50 \times 1.00+0.50 \times 1.00=1.00 \text { bit }
$$

The value of 1 here represents the 1 bit of information that is gained when learning of a suspect's guilt or innocence.

An unbalanced set, say $80 \%$ guilty suspects and $20 \%$ innocent suspects, supports a higher level of certainty. Specifically, in this case we are justified in being $80 \%$ certain in our ability to correctly judge guilt or innocence. Entropy measures uncertainty, so this higher certainty corresponds to lower entropy. Specifically, with this distribution, the best we can do is judge that every suspect has an 80-20 chance of being guilty or innocent. In other words, we pretty strongly expect that each suspect is guilty, and this expectation is usually confirmed. Thus, relative to a 50-50 set, judgment error decreases. Specifically,

$$
\begin{gathered}
I(g)=\log _{2}(1 / p(g))=\log _{2}(1 / 0.80)=0.32 \text { bits } \\
I(i)=\log _{2}(1 / p(i))=\log _{2}(1 / 0.20)=2.32 \text { bits } \\
H(Y)=p(g) I(g)+p(i) I(i)=0.80 \times 0.32+0.20 \times 2.32=0.72 \text { bits } .
\end{gathered}
$$

Notice that judgment error has actually increased for innocent suspects relative to the 50-50 set of suspects (from 1 to 2.32 bits). This increase occurred because the innocent suspects in the 8020 set had the bad luck of being mixed in with mostly guilty suspects, creating the misleading 
impression that they were probably guilty. However, because innocent suspects are rare, the average judgment error has decreased relative to the 50-50 set (from 1 to 0.72 bits).

The same reasoning follows for a set $20 \%$ guilty suspects and $80 \%$ innocent suspects. Again, with this set we are justified in being $80 \%$ certain in our ability to correctly judge guilt or innocence, and the increase in certainty corresponds to a lower entropy. Specifically, the best we can do is judge a $20-80$ chance that each suspect is guilty or innocent, and the average judgment error is

$$
\begin{gathered}
I(g)=\log _{2}(1 / p(g))=\log _{2}(1 / 0.20)=2.32 \text { bits, } \\
I(i)=\log _{2}(1 / p(i))=\log _{2}(1 / 0.80)=0.32 \text { bits, } \\
H(Y)=p(g) I(g)+p(i) I(i)=0.20 \times 2.32+0.80 \times 0.32=0.72 \text { bits. }
\end{gathered}
$$

In colloquial terms, judgment error has decreased relative to the 50-50 set because we expect that each suspect is innocent, and this expectation is usually confirmed.

In the most extreme case, in which a set of suspects all belong to the same guilt category, we can make perfect judgments, and learning the guilt category provides no new information. For example, in a set of only guilty suspects, we expect a $100 \%$ chance of guilt for each suspect, and this expectation is always confirmed, yielding zero judgment error,

$$
\begin{gathered}
I(g)=\log _{2}(1 / p(g))=\log _{2}(1 / 1)=0 \text { bits } \\
H(Y)=p(g) I(g)=1 \times 0=0 \text { bits. }
\end{gathered}
$$

Note that innocent suspects are eliminated from the entropy calculation because there are none in the set. Likewise, for a set of only innocent suspects, we expect a $100 \%$ chance of innocence for each suspect, and this expectation is always confirmed, again yielding zero judgement error,

$$
\begin{gathered}
I(i)=\log _{2}(1 / p(i))=\log _{2}(1 / 1)=0 \text { bits, } \\
H(Y)=p(i) I(i)=1 \times 0=0 \text { bits. }
\end{gathered}
$$


In both cases, an entropy of zero corresponds to $100 \%$ certainty in our ability to correctly specify guilt or innocence.

\section{Information Gain}

Our goal is to measure the evidentiary value of eyewitness identification procedures, so we are concerned with judgments that represent the prior and posterior probabilities of guilt in a Bayesian analysis informed by the identification responses. To illustrate this goal, we will present some examples of using entropy to assess the show-up data in Table 1. The prior distribution in this hypothetical show-up data set is characterized by a $50 \%$ probability of guilt and a $50 \%$ probability of innocence, corresponding to an entropy of $1 \mathrm{bit}$, as calculated above. This entropy value gives the average judgment error, i.e., the average surprisal, achieved after judging the guilt status of all of the show-up trials in the example data set without knowledge of the witness responses.

To move to the other extreme, imagine that a witness makes Response $x$, and that this response uniquely determines a suspect's guilt or innocence. For example, imagine that witnesses in the example show-up experiment were perfectly accurate in their identification responses, i.e., every single identification came from a show-up with a guilty suspect. In this situation, learning that the witness identified the suspect on a particular show-up trial changes the distribution of uncertainty about guilt to $p(g \mid x)=1$ and $p(i \mid x)=0$, and entropy goes to zero as calculated previously. Again, this value represents the average judgment error (surprisal) across an entire set, but here the evidence, in the form of identifications, isolated a set composed entirely of guilty suspects, facilitating perfect judgments. The same would be true for evidence that uniquely determines innocence, for example, if witnesses in the show-up data set were perfectly accurate in their rejection responses. 
Wells and Lindsay (1980) used the term "information gain" to describe the change in uncertainty about guilt or innocence from the prior distribution to the posterior distribution associated with a particular witness response. We will adopt the same definition, but we will measure uncertainty on a Shannon surprisal scale instead of a probability scale. Thus, in the current context, information gain is the reduction in entropy achieved by observing a particular witness response. ${ }^{3}$ Considering the last example, entropy changed from 1 bit before observing that the witness made Response $x$ to 0 bits after observing $x$. Thus, we have gained $H(Y)-H(Y \mid$ $x)=1-0=1$ bit of information from the witness response. This value of 1 bit is a good reference point to keep in mind for interpreting information gain values. For the question of a suspect's guilt or innocence (or any other variable with two outcomes), the value 1 represents the amount of information needed to go from complete uncertainty to full knowledge of the true state of affairs. In other words, obtaining 1 bit of information requires access to perfect evidence. ${ }^{4}$ Of course, witnesses in our hypothetical show-up experiment did not provide perfect evidence, as they sometimes made errors in both their identification and rejection responses. Nevertheless, information gain allows us to quantify the value of the imperfect evidence provided by witness responses. We will begin with the 10-second viewing condition. As calculated previously, after an identification, the probability of guilt changed to $p(g \mid i d)=0.89$ and the probability of innocence changed to $p(i \mid i d)=0.11$. The associated surprisals are

$$
I(g \mid i d)=\log _{2}(1 / p(g \mid i d))=\log _{2}(1 / 0.89)=0.17 \text { bits }
$$

\footnotetext{
${ }^{3}$ The term "information gain" is sometimes used to denote the mutual information between two random variables, a quantity that we call "expected information gain" (e.g., Quinlan, 1986). For continuity with previous eyewitness studies (e.g., Wells \& Lindsay, 1980), we will use "information gain" when referring to a particular witness response and "expected information gain" when referring to the average information gain across a set of identification attempts.

${ }^{4}$ In the general case, with $n$ possible outcomes, the maximum amount of information gained relative to complete uncertainty is given by $\log _{2}(n)$.
} 
and

$$
I(i \mid i d)=\log _{2}(1 / p(i \mid i d))=\log _{2}(1 / 0.11)=3.17 \text { bits. }
$$

Entropy $^{5}$ can then be calculated as

$$
\begin{gathered}
H(Y \mid i d)=p(g \mid i d) I(g \mid i d)+p(i \mid i d) I(i \mid i d)= \\
0.89 \times 0.17+0.11 \times 3.17=0.50 \text { bits } .
\end{gathered}
$$

The entropy here represents the average judgment error across the entire set of show-ups with a suspect identification from the 10-second condition. Although entropy has not dropped to zero, as in the case of perfect evidence, it has decreased to 0.50 from the initial value of 1 associated with the prior distribution. This decrease was achieved because the set of show-up trials with a witness identification primarily contains trials with a guilty suspect, decreasing uncertainty about guilt.

Likewise, for rejections in the 10 -second condition, $p(g \mid r e j)=0.18$ and $p(i \mid r e j)=0.82$,

so,

$$
I(g \mid r e j)=\log _{2}(1 / p(g \mid r e j))=\log _{2}(1 / 0.18)=2.46 \text { bits }
$$

and

$$
I(i \mid r e j)=\log _{2}(1 / p(i \mid r e j))=\log _{2}(1 / 0.82)=0.29 \text { bits. }
$$

Entropy can then be calculated as

$$
\begin{gathered}
H(Y \mid r e j)=p(g \mid r e j) I(g \mid r e j)+p(i \mid r e j) I(i \mid r e j)= \\
0.18 \times 2.46+0.82 \times 0.29=0.68 \text { bits }
\end{gathered}
$$

for an information gain of $H(Y)-H(Y \mid r e j)=1-0.68=0.32$ bits. In this situation, less information about guilt is gained from a rejection, 0.32 bits, than from an identification, 0.50 bits, because identifications isolate show-up trials from a single guilt category more effectively

\footnotetext{
${ }^{5}$ Entropy conditioned on a particular value, i.e., $H(Y \mid X=x)$, is called the specific conditional entropy.
} 
than rejections. That is, identifications isolate a set with an 89-11 balance of guilty-to-innocent suspects, whereas rejections isolate an $18-82$ set. This means that there is more uncertainty about guilt after a rejection than after an identification, producing higher average judgment error.

\section{Expected Information Gain (EIG)}

One more step is required to reach our goal: Averaging information gain across all identification attempts to define Expected Information Gain (EIG). ${ }^{6}$ The result indexes the overall evidentiary value of an identification procedure when witness responses vary from one attempt to the next, a condition that holds for any procedure adopted in real investigations. Because the Shannon surprisal scale is additive over uncertainty, as discussed previously, this step is the point at which measuring uncertainty on this scale becomes crucial. Just as it was very useful for Shannon (1948) to develop a measure that validly expressed average uncertainty per unit time (p. 13), it is useful for eyewitness researchers to have a measure that validly expresses average uncertainty per identification attempt.

For the 10-second condition summarized in Table 1, we have calculated the amount of information gained from both identifications, 0.50 bits, and rejections, 0.32 bits. The final step is to take a weighted average to determine the EIG across all identification attempts. From Table 1, $45 \%$ of responses in this condition are identifications and 55\% of responses are rejections. Thus, the expected information gain is given by

$$
\begin{aligned}
E I G= & p(i d) \times(H(Y)-H(Y \mid i d))+p(r e j) \times(H(Y)-H(Y \mid r e j))= \\
& 0.45 \times(1-0.50)+0.55 \times(1-0.68)=0.40 \text { bits } .
\end{aligned}
$$

\footnotetext{
${ }^{6}$ Expected information gain is often called "mutual information" (Cover \& Thomas, 2007) or sometimes simply "information gain" (Quinlan, 1986). Again, we adopt the term "expected information gain" in an attempt to clarify the link between this measure and previous measures discussed by eyewitness memory researchers (e.g., Wells \& Lindsay, 1980).
} 
That is, in this condition, we expect to gain 0.40 bits of information after learning a witness response. This number can be compared to the EIG score from any other procedure to investigate how system and/or estimator variables impact the evidentiary value of witness reports. For example, we can compare this value to the EIG value from the 30-second viewing condition, which we calculate next.

The 30-second condition also had the same number of show-up attempts with a guilty suspect and an innocent suspect, so as before, the entropy for the prior distribution is 1 bit. Out of the show-ups that led to an identification of the suspect, $95 \%$ had a guilty suspect and $5 \%$ had an innocent suspect, so the entropy for the posterior distribution is

$$
\begin{gathered}
I(g)=\log _{2}(1 / p(g))=\log _{2}(1 / 0.95)=0.07 \text { bits } \\
I(i)=\log _{2}(1 / p(i))=\log _{2}(1 / 0.05)=4.32 \text { bits } \\
H(Y)=p(g) I(g)+p(i) I(i)=0.95 \times 0.07+0.05 \times 4.32=0.28 \text { bits }
\end{gathered}
$$

representing an information gain of $1-0.28=0.72$ bits. Out of the show-ups that led to a rejection, $9.5 \%$ had a guilty suspect and $91.5 \%$ had an innocent suspect, so the entropy for the posterior distribution is

$$
\begin{gathered}
I(g)=\log _{2}(1 / p(g))=\log _{2}(1 / 0.095)=3.40 \text { bits } \\
I(i)=\log _{2}(1 / p(i))=\log _{2}(1 / 0.915)=0.13 \text { bits } \\
H(Y)=p(g) I(g)+p(i) I(i)=0.095 \times 3.40+0.915 \times 0.13=0.44 \text { bits, }
\end{gathered}
$$

representing an information gain of $1-0.44=0.56$ bits. Overall, witnesses identified the suspect $47.5 \%$ of the time and rejected the suspect $52.5 \%$ of the time, so the EIG value for the procedure as a whole is

$$
\begin{gathered}
E I G=p(i d) \times(H(Y)-H(Y \mid i d))+p(r e j) \times(H(Y)-H(Y \mid r e j))= \\
0.475 \times(1-0.28)+0.525 \times(1-0.44)=0.64 .
\end{gathered}
$$


Now we can compare the 10 -second and 30 -second conditions, revealing that viewing time had a substantial impact on the evidentiary value of witness responses, with an EIG of 0.4 for the 10 -second condition and 0.64 for the 30 -second condition. This result is consistent with the conclusion we made in the previous section based on the prior and posterior probabilities for identifications and rejections, but the EIG analysis combines all of the relevant factors to give a single summary value on a scale that is appropriate for expressing average uncertainty across multiple outcomes. Critically, the demonstrations below show that EIG often reveals surprising results and can be used to address research questions that are out of reach for existing metrics.

\section{Expected Probability Gain (EPG)}

Many readers will have little or no experience working with Shannon surprisal as a unit of measurement. To facilitate the interpretation of overall evidentiary value, we translate the change in entropy back to the corresponding change in the probability parameter of a Bernoulli distribution representing uncertainty about guilt, yielding a value that we will call the Expected Probability Gain $(E P G)$. EPG values do not indicate the change in the probability of guilt or the probability of innocence; instead, they indicate the change in the probability that guilt or innocence can be judged accurately, or equivalently, the change in the level of certainty about guilt or innocence on a probability scale. For example, both a $90 \%$ probability of guilt and a $10 \%$ probability of guilt correspond to $90 \%$ certainty, because in the former case judgments of guilt have a $90 \%$ chance of being correct and in the latter case judgments of innocence have a $90 \%$ chance of being correct.

Figure 3 shows the relationship between probability gain and information gain for a single type of response (e.g., a suspect identification or a rejection) when the prior probability of guilt is $50 \%$, as in the hypothetical show-up experiment. We discuss how to compute the solid- 
black curve in the EPG Equations section below; for now we just provide the results. A probability gain of zero means that the identification procedure had no evidentiary value, i.e., the certainty about guilt is $50 \%$ in both the prior and the posterior distribution. A probability gain of $50 \%$ means that the identification procedure provided perfect evidence, i.e., the 50\% certainty for the prior distribution increased to $100 \%$ certainty for the posterior distribution. The $10-$ second condition's EIG of 0.4 corresponds to roughly a 35\% EPG, and the 30-second condition's EIG of 0.64 corresponds to a 43\% EPG. Thus, EPG translates EIG to a more familiar scale, which, in this case, reveals that considering the witness responses substantially increases certainty about guilt category, especially for the longer viewing time.

Our EPG measure is similar to a measure that other eyewitness researchers have called “information gain” (Wells \& Lindsay, 1980; Wells \& Olson, 2002; Wells \& Turtle, 1986; Wells, Yang, \& Smalarz, 2015). Given the potential for confusion, we will now explain the differences between these two measures. First, of course, we use the term "probability gain" to distinguish this measure from "information gain" as defined as a reduction in entropy (Quinlan, 1986). Second, EPG is a composite value over a set of identification attempts with variation in witness responses from one attempt to the next, whereas previous papers separately analyzed individual response types (e.g., identifications and rejections have separate values: Wells \& Lindsay, 1980; Wells, Yang, \& Smalarz, 2015). So, for example, the 10-second condition's EPG of 35\% means that, on average, witness responses increased certainty about guilt by 35 percentage points, representing a change from $50 \%$ to $85 \%$ certainty about the true guilt category. Again, these certainty values are indifferent to which guilt category is supported - evidence that supports $75 \%$ certainty that a suspect is guilty is equivalent to evidence that supports $75 \%$ certainty that a suspect is innocent. The probabilities cannot be directly averaged, because the probability scale 
is multiplicative over uncertainty, not additive. Averaging on the surprisal scale and then translating back to probability, as was done to calculate EPG, solves this problem.

That last point is the main motivation for our use of the surprisal scale, so we illustrate it with an example that further illustrates the difference between the current approach and previous approaches (Wells \& Lindsay, 1980). Using Figure 3 as a reference, we contrast two simple sets of identification results to show that averaging probabilities distorts conclusions. For both procedures, we keep things simple by assuming a 50\% prior probability of guilt. We also simplify the example by averaging across conditions within the same response type, but the averaging artifacts also arise in the more common case of averaging across different response types within a condition.

Assume that in Procedure A, 75\% of identified suspects are guilty. This situation represents an increase in certainty about guilt of 0.25 on the probability scale $(0.75-0.50)$ and 0.19 on the surprisal scale (the triangle in Figure 3). Procedure B splits identification attempts evenly between two conditions, one that produced low accuracy and another that produced high accuracy. For the low-accuracy condition, 55\% of identified suspects were guilty, representing an increase in certainty of 0.05 on the probability scale and 0.01 on the surprisal scale (the lowerleft $\mathrm{x}$ in Figure 3). For the high-accuracy conditions, 95\% of identified suspects were guilty, representing an increase in certainty of 0.45 on the probability scale and 0.71 on the surprisal scale (the upper-right circle in Figure 3). The gray lines and the combined circle-x point in Figure 3 show the result of averaging the change in certainty across the low- and high-accuracy conditions on both scales (probability and information gain). The across-condition average change in certainty is 0.25 on the probability scale, $(0.05+0.45) / 2=0.25$, suggesting that Procedure A and Procedure B produce the same overall change in certainty about guilt across all 
identification attempts. In contrast, the across-condition average change in certainty is 0.36 on the surprisal scale, $(0.01+0.71) / 2=0.36$, suggesting the Procedure B produces a larger overall increase in certainty compared to Procedure A (.19). The average based on surprisal is the correct one, because uncertainty is additive on this scale (Shannon, 1948). Directly averaging the probabilities produces a different, and misleading, answer due to averaging artifacts on the nonadditive probability scale.

This example shows that it is critical that the averages are taken on the surprisal scale even if the probability scale is used for interpretation. For previous applications that evaluated a single type of response at a time (Wells \& Lindsay, 1980; Wells \& Olson, 2002; Wells \& Turtle, 1986; Wells et al., 2015), the two scales will always agree on the relative ranking of procedures; that is, they will agree on which procedure best reduces uncertainty about guilt when a witness makes that particular response. In other words, the function relating the two scales is monotonic, as shown in Figure 3). However, the function relating the two scales is not linear, so averages on the probability scale do not accurately characterize average uncertainty and can even support the wrong conclusion about the relationship between two procedures.

\section{Conceptual Summary of Information Theory and Identifications}

This section explained our proposed method for defining the evidentiary value of eyewitness identification procedures. We used a hypothetical show-up data set as a simple example, where the only procedural difference investigated was how long the witness viewed the suspect, 10 seconds or 30 seconds. We evaluated the evidentiary value of witness responses in each viewing-time condition by combining the principles of probabilistic inference (McElreath, 2016) with foundational concepts in information theory (Shannon, 1948). 
As noted by previous eyewitness researchers (e.g., Wells \& Lindsay, 1980), Bayesian inference defines how a particular identification response changes the probability that a suspect is guilty from a prior to a posterior distribution representing the state of uncertainty before and after the witness response is observed, respectively. Contrasting the 10-second and 30-second conditions revealed that identifications were stronger evidence of guilt and rejections were stronger evidence of innocence in the latter condition, a direct consequence of the fact that witness responses were more accurate with longer viewing time (see Table 1). The critical take-away from the Bayesian example is that the change in certainty about guilt or innocence is determined by the strength of evidence available, and thus it is a meaningful measure of the evidentiary value of a particular identification response, as first noted by Wells and Lindsay (1980).

Identification procedures are used across many separate identification attempts, and witness responses will vary from one attempt to the next. Thus, measuring the full evidentiary value of a procedure necessitates a method for combining across possible responses. This goal is facilitated by switching from measuring uncertainty on the probability scale to measuring uncertainty on the Shannon surprisal scale (Shannon, 1948). We discussed Shannon surprisal as a measure of the degree of error in a probabilistic judgment. In short, if a judgment assigns a low probability to the true state of affairs, then that decision maker would be very surprised to learn the truth, signaling that they judged poorly (high error). If a judgment assigns a high probability to the true state of affairs, then the decision maker would not be very surprised to learn the truth, signaling that they judged effectively (low error). We noted that this measure of judgment success is the only one that is fully consistent with Bayesian inference (Bickel, 2007), and we demonstrated an important facet of this consistency by showing that Shannon surprisal provides a strictly proper scoring rule for measuring judgment error (Savage, 1971; Winkler, 1969). This property means 
that the best strategy for minimizing judgment error is to express a level of uncertainty consistent with the true risk of making an incorrect judgment (see Figure 2).

Combining these key innovations from probability and information theory produces a theoretically grounded method for measuring evidentiary value (e.g., Ramos \& GanzalezRodriguez, 2008). The change in average Shannon surprisal, or entropy, from the prior to the posterior distribution in a Bayesian analysis defines the information gain, or the reduction in uncertainty about guilt or innocence. Because this measure puts uncertainty on an additive scale, we can simply average across all identification attempts to define overall evidentiary value with an Expected Information Gain (EIG) score.

In brief, applying Bayesian inference ensures that the level of certainty expressed in judgments of guilt or innocence matches the true uncertainty supported by the evidence available (i.e., the probability of correctly specifying guilt or innocence given the evidence), and information gain quantifies the degree to which a given piece of evidence decreases uncertainty when it is properly interpreted. EIG considers the information gain of all possible witness responses and how often these responses are obtained to characterize the full evidentiary value of an identification procedure. That is, EIG determines how much the procedure reduces uncertainty about guilt or innocence on average across identification attempts. Expected probability gain (EPG) translates EIG from the Shannon surprisal scale of uncertainty back to the probability scale to aid in interpretation (see Figure 3). Thus, these measures allow eyewitness researchers to directly compare different identification procedures with a metric that considers every factor relevant to evidentiary value. Higher EIG and EPG values indicate procedures that are more effective in reducing uncertainty about guilt or innocence. 


\section{EIG and EPG Equations for Eyewitness Researchers}

In this section, we compile all of the equations needed to calculate EIG and EPG for an eyewitness memory data set for easier reference. The equations reported here are designed for general application across a variety of different data sets regardless of the identification format (e.g., lineup or show-up) and the potential witness responses (e.g., responses with or without confidence ratings). To facilitate comparisons across data sets and conditions, the equations give the EIG value associated with a 50\% prior probability of guilt even when there are a higher or lower proportion of identification attempts involving guilty suspects. Using a prior probability other than $50 \%$ reduces the EIG value, with a larger reduction for prior probabilities that are closer to 0 or $100 \%$. For example, if all of the suspects in a data set are guilty (e.g., Schooler \& Engstler-Schooler, 1990), then the prior distribution has an entropy of zero and it is impossible to gain any information from the witness responses. Standardizing EIG values to a $50 \%$ prior probability of guilt defines the effectiveness of an identification procedure when it is the only source of information about guilt or innocence. Although a 50\% prior probability of guilt cannot be assumed in every application of an identification procedure, of course, the Demonstrations Exploring EIG section below establishes that the identification procedures that optimize EIG under a 50\% prior probability are near-optimal for any actual prior probability.

This section does not have a conceptual summary, so readers who prefer to skip the EIG equations should advance to Demonstrations Exploring EIG.

\section{EIG Equations}

Throughout, we use $Y$ to refer to the distribution of guilt, $g$, or innocence, $i$. We use $X$ to refer to the distribution of witness responses with $x$ identifying a particular response; for example, $x$ could be an identification, $i d$, or rejection, $r e j$. For example, $H(Y \mid X=i d)$ is the 
entropy of the distribution of guilt and innocence given that the response was an identification. Where clear in context, we simplify as $H(Y \mid i d)$. The possible responses in $X$ can vary across situations, as will be shown below. EIG is a function of both $Y$ and $X$, but, because these values are always provided by context, we do not include them in the notation.

For this calculation of EIG, the prior distribution is always characterized by $p(g)=0.50$, the prior probability of guilt, and $p(i)=0.50$, the prior probability of innocence. The overall probability that a witness will make Response $x$, e.g., an identification or rejection (but not restricted to these responses), is given by

$$
p(x)=0.50 \times p(x \mid g)+0.50 \times p(x \mid i),
$$

where $p(x \mid g)$ and $\mathrm{p}(x \mid i)$ are the observed likelihoods of Response $x$ for guilty and innocent suspects, respectively. In short, Equation 38 provides the probability of each response in a pool of identification attempts with half guilty and half innocent suspects. The posterior probability of guilt, $p(g \mid x)$, for each possible witness response is given by

$$
p(g \mid x)=(0.50 \times p(x \mid g)) /(0.50 \times p(x \mid g)+0.50 \times p(x \mid i)),
$$

and the posterior probability of innocence is given by

$$
p(i \mid x)=1-p(g \mid x)
$$

Equations 39 and 40 define the posterior distributions associated with each witness response in a pool of identification attempts with half guilty and half innocent suspects. Notice that these equations only rely on the likelihood of observing a given response for identification attempts with guilty and innocent suspects. So, for example, two data sets that have the same likelihoods will get the same EIG value even if one data set had an exactly equal number of guilty-suspect and innocent-suspect trials and the other happened to have mostly guilty-suspect trials. These 
sorts of procedural details are not relevant for determining the effectiveness of an identification procedure for real investigations, so it is helpful to use a version of EIG that ignores them.

Given the assumed $50 \%$ prior probability of guilt, the entropy for the prior distribution of uncertainty about guilt is given by $H(Y)=1$, as calculated in Equation 13. The entropy for the posterior distribution of uncertainty associated with witness Response $x$ is given by

$$
H(Y \mid x)=p(g \mid x) \log _{2}(1 / p(g \mid x))+p(i \mid x) \log _{2}(1 / p(i \mid x))
$$

and the information gain, $I G$, is given by

$$
I G(x)=H(Y)-H(Y \mid x)=1-H(Y \mid x)
$$

Finally, the expected information gain is given by

$$
E I G=\Sigma p(x) I G(x)
$$

where the sum is over all possible responses.

\section{EPG Equations}

As discussed previously, Expected Probability Gain (EPG) is a useful supplement for interpreting EIG that converts certainty/uncertainty back to a probability scale. Specifically, EPG is the average change in certainty about a suspect's guilt across a full set of identification attempts. To avoid averaging artifacts, the averaging occurs on the surprisal scale (see Figure 3), and then the result is converted to the probability scale.

Unfortunately, there is no closed form equation for translating the entropy of a dichotomous variable (like guilt or innocence) to a probability. EPG values can be found either with a search algorithm or with the following approximation based on the formula reported by Zhou, Cheng, He, \& Matsumodo (2014):

$$
E P G \approx .5-\left(2^{0.6794 \times(1-E I G)^{0.7244}}-2^{-0.1357 \times(1-E I G)^{21.8026}}\right)^{1.9920}
$$


When calculated under the assumptions imposed here, an EPG value of $50 \%$ means that the witness responses perfectly discriminated the guilty and innocent suspects, such that the average certainty about guilt increased from $50 \%$ to $100 \%$. An EPG value of zero means that the witness responses had no evidentiary value, in that the average certainty about guilt was $50 \%$ both before and after considering the witness responses.

\section{Demonstrations Exploring EIG}

The previous sections developed EIG as a measure for comparing eyewitness identification procedures. In the current section, we present theoretical demonstrations designed to highlight key properties of EIG and illustrate how it differs from existing measures. Specifically, we contrast EIG with diagnosticity ratios (Wells \& Lindsay, 1980), area under the ROC (Mickes et al., 2012), deviation from perfect performance (Smith et al., 2019), and expected utility (Ceci \& Freidman, 2000). In each case, we demonstrate that EIG provides a more complete assessment of the effectiveness of an identification procedure when the goal is using the witness responses to improve judgments of guilt or innocence. Indeed, EIG considers all factors relevant to a procedure's evidentiary value. The final demonstration shows how EIG can define the evidentiary value of having access to continuous predictors of witness accuracy, using the response time (RT) of identification decisions as an example. This demonstration shows that EIG can be used to address important research questions that are not accessible to the existing measures.

Readers who are not interested in quantitative details can skip to the Conceptual Summary of EIG Demonstrations section that follows the demonstrations. 


\section{Simulating Data with Signal Detection Models}

Signal detection models are used to generate example data sets for most of the following demonstrations (Macmillan \& Creelman, 2005). Signal detection theory (SDT) is probably the most broadly applied mathematical model in psychology (Wixted, 2020), and signal detection models have proven to be quite useful for analyzing identification data (e.g., Wixted et al., 2018). Using simulated data from a well-validated model allows us to isolate individual processes that distinguish EIG from the other measures, while, at the same time, ensuring that we are working with realistic data patterns.

The signal detection model assumes that witnesses base their identification responses on the match between the suspect and their memory for the culprit, with separate distributions of match strength for identification attempts with a guilty suspect and identification attempts with an innocent suspect (for detailed model descriptions, see Cohen et al., 2020; Wixted et al., 2016). Match strength tends to be higher for identification attempts with a guilty suspect, of course, but match strength varies across attempts for both guilty and innocent suspects. This variation is driven by many factors, including factors that affect how well witnesses remember the culprit (e.g., whether or not they got a clear view), factors that affect how much the appearance of a guilty suspect changes from the witnessed crime to the identification attempt (e.g., whether or not the culprit grew a beard), and factors that affect how much an innocent suspect looks like the culprit (e.g., whether or not the culprit and innocent suspect happen to share a lot of facial features). We assumed that variability in match strength was equal for identification attempts with guilty and innocent suspects (Wixted et al., 2016).

The collective ability of witnesses to discriminate guilty and innocent suspects is represented by $d^{\prime}$, the standardized distance between the means of the memory match 
distributions for innocent and guilty suspects. A $d^{\prime}$ of 0 means that the witness cannot discriminate between guilty and innocent suspects. As $d^{\prime}$ increases, the match strength distributions for guilty and innocent suspects become increasingly distinct, representing an improved ability to distinguish guilty and innocent suspects.

The response criterion is the cutoff for how well the suspect must match the witness' memory of the culprit to justify an identification response. A low, or liberal, criterion means that even a relatively poor match justifies an identification response. In contrast, a high, or conservative, criterion means that a strong match is needed to justify an identification response.

Variations of this basic signal detection model are described in each demonstration. The final demonstration uses the diffusion model (Ratcliff, 1978), which implements a dynamic version of the signal detection process in which the match strength varies over time within a single decision (for more information, see Ratcliff \& McKoon, 2008). Although this model has not been applied to lineup procedures, to our knowledge, it has been extensively validated for two-choice decision tasks that match the form of a show-up procedure (i.e., see a single stimulus and decide which of two stimulus classes it belongs to; Ratcliff \& McKoon, 2008). In fact, if Signal Detection Theory is the most broadly applied mathematical model in psychology (Wixted, 2020), the diffusion model is a strong candidate for the second-most broadly applied (Wagenmakers, 2009).

\section{Demonstration 1: EIG versus Diagnosticity Ratios}

\section{The Diagnosticity Ratio}

One popular approach to evaluating identification procedures is to compare diagnosticity ratios to assess the probative value of witness responses (e.g., Clark, 2012; Price \& Fitzgerald, 2016; Wells \& Lindsay, 1980; Wells \& Turtle, 1986; Wells, Yang, \& Smalarz, 2015). The 
diagnosticity ratio for a given witness response is calculated by dividing the probability of observing the response for a guilty suspect by the probability of observing the response for an innocent suspect. Although diagnosticity ratios can be calculated for any type of response, we will mostly focus on the diagnosticity of suspect identifications, as this is often the outcome of greatest concern to investigators (Wells \& Turtle, 1986); indeed, some investigators report the unfortunate practice of failing to record identification attempts that result in non-identifications (Police Executive Research Forum, 2013).

The hypothetical show-up experiment summarized in Table 1 can serve as a simple example of using diagnosticity ratios to evaluate an identification procedure. For the 10 -second condition, $80 \%$ of guilty suspects and $10 \%$ of innocent suspects were identified by a witness, so the diagnosticity ratio is $8(0.80 / 0.10)$. For the 30 -second condition, $90 \%$ of guilty suspects and $5 \%$ of innocent suspects were identified, for a diagnosticity ratio of $18(0.90 / 0.05)$. Higher ratios indicate that identifications are stronger evidence of guilt, so a high diagnosticity ratio is certainly a desirable quality for an identification procedure. In this case, diagnosticity ratios suggest that increased viewing time enhances the evidentiary value of witness responses, the same conclusion we reached earlier based on EIG. The current demonstration explores a scenario in which these measures support different conclusions.

Some eyewitness memory researchers have noted that the diagnosticity ratio is an incomplete measure of the effectiveness of identification procedures (Mickes et al., 2012; Rotello \& Chen, 2016; Wixted \& Mickes, 2012). Wixted and Mickes (2012) and Rotello and Chen (2016) provided a clear demonstration of this by exploring how the diagnosticity ratio is affected by changes in response bias, that is, a witness' overall willingness to make an identification response. This point is important, so we explored the effect of changes in response 
bias to illustrate some critical differences between diagnosticity ratios and EIG. For simplicity, we considered a show-up procedure and used a signal detection model to precisely and uniquely manipulate response bias while ensuring realistic patterns of simulated data.

\section{The Effect of Criterion Shifts}

Using a signal detection model, we varied the response criterion along a full range from very low (liberal) values to very high (conservative) values. This variation represents the effect of policy choices that influence witness conservativeness via system variables, for example, whether or not the witnesses is warned that the suspect might not be the culprit (Wixted \& Mickes, 2012). Of course, these policies could never achieve the precise control over witness conservativeness represented by the range of criterion values in our demonstration, but that is exactly why it is instructive to use a "sanitized," model-based example. We assume a reasonable level of witness accuracy, $d^{\prime}=1.5$. For our purposes, it was convenient to make zero the reference point for an unbiased criterion, so we set the position of the match-strength distributions to $d^{\prime} / 2$ for attempts with guilty suspects and $-d^{\prime} / 2$ for attempts with innocent suspects (Macmillan \& Creelman, 2005). For our first simulation, we used a model in which half of the witnesses saw a guilty suspect and half saw an innocent suspect.

Figure 4 summarizes the results of the criterion demonstration. Panel A shows how changing the criterion changes identification rates for guilty and innocent suspects (dashed and dotted lines, respectively). Guilty suspects are more likely to be identified than innocent suspects, and identification rates for both guilty and innocent suspects decrease with increasingly conservative responding (higher criterion values).

For each criterion value, Panel B shows the associated diagnosticity ratio, which is found by dividing the height of the dashed line by the height of the dotted line at a given $x$-axis value in 
Panel A. This panel shows the pattern noted by Wixted and Mickes (2012; see also Rotello \& Chen, 2016): When the criterion is liberal, a high proportion of identifications are made overall and the diagnosticity ratio is low because the numerator cannot exceed 1 and the denominator is close to 1 . When the criterion is conservative, a low proportion of identifications are made overall and the diagnosticity ratio becomes arbitrarily large. Thus, using the diagnosticity of identifications to measure evidentiary value suggests that administrators should adopt procedures that make witnesses as conservative as possible; for example, this result suggests that it would be a good idea to tell witnesses there is a very low chance that the suspect was the actual culprit (Wixted \& Mickes, 2012).

The problem with the diagnosticity ratio measure is that it only considers the evidentiary value of a single response, ignoring both the overall probability of obtaining the response and the evidentiary value of all alternative responses. Intuitively, the fact that identifications are strong evidence of guilt is not very useful if identifications almost never happen, so just getting witnesses to respond as conservatively as possible is probably not a good strategy. Moreover, conservative responding makes rejections more ambiguous, i.e., they cannot be interpreted as strong evidence for innocence given that witnesses fail to identify many of the guilty suspects in addition to many of the innocent suspects.

The EIG measure, in contrast, combines all relevant factors; namely, the probability and the evidentiary value of every possible identification response. (It also measures evidentiary value on a scale with better measurement properties, as we demonstrate shortly.) The right-hand panels of Figure 4 show how the factors influencing EIG work together for the criterion example.

Panel C of Figure 4 shows the overall probability of identifications (blue curve) and rejections (red curve) across different possible values for the response criterion, and Panel D 
shows the associated information gain for identifications and rejections. As before, the information gain values are the difference in average Shannon surprisal, $I$, from the prior to posterior distribution of uncertainty about the suspect's guilt category (or equivalently, the difference in the entropy). The information gain for identifications increases as responding becomes more conservative. With very liberal responding, witnesses almost always identify the suspect, and information gain for identifications is low because there is almost as much uncertainty about guilt after an identification as before. As the criterion becomes more conservative, identifications become rarer and more specific to identification attempts that involved a guilty suspect (innocent suspects rarely have a strong match to the culprit). This criterion shift increases information gain via the same mechanism that increases the diagnosticity ratio, but the information gain measure approaches a value of 1 rather than becoming an arbitrarily high value (recall that 1 bit represents going from complete uncertainty to complete certainty about guilt or innocence). Moreover, unlike diagnosticity, EIG considers all possible lineup responses, and Panel D shows that more conservative responding decreases the information gain for rejections.

Panel E shows EIG, which, of course, is a product of the information gain for each possible response (Panel D) and its corresponding probability (Panel C). Generally, comparing Panels A and B to Panels C and D in Figure 4 illustrates that EIG considers more aspects of performance than the diagnosticity ratio; indeed, EIG considers every aspect of performance that is relevant to the informational value of identifications as an evidence source. In this example, EIG is highest for a criterion value of 0 , representing unbiased responding.

The patterns in Panels C and D provide an explanation for why EIG prioritizes unbiased responding: Response biases simultaneously make one type of response more common overall 
and degrade the evidentiary value of that response, resulting in a lower EIG value. Thus, whereas optimizing the diagnosticity of identifications motivates policies that encourage witnesses to respond as conservatively as possible, EIG suggests that the best policy is to encourage unbiased responding. If the goal of the identification procedure is to provide evidence that supports effective judgments about which suspects are guilty and which are innocent, then the EIG prescription is the right one.

We have considered the simplest version of diagnosticity ratio analysis in which only identification responses are considered. Some authors have gone farther by assessing diagnosticity for both identifications and rejections (e.g., Wells \& Lindsay, 1980), and readers may wonder if we could combine diagnosticity ratios across these responses to characterize overall evidentiary value. The answer is no, because this would introduce the same sort of averaging artifacts that we noted earlier for the probability scale (Figure 3). Figure 5 shows how information gain is related to both diagnosticity ratios and log diagnosticity ratios. Neither measure is linearly related to information gain, which means that neither measure puts uncertainty on an additive scale, as does Shannon surprisal (Shannon, 1948). Thus, summing or averaging (log) diagnosticity ratios cannot accurately characterize the total or average uncertainty about guilt. Thus, these measures are not appropriate for measuring the overall evidentiary value of a procedure across multiple identification attempts.

\section{A Note on Prior Probability}

The criterion-setting example provides an opportunity to highlight an important point: In some situations, changing the prior probability of guilt can change the procedure that produces 
the highest EIG. ${ }^{7}$ Figure 6 shows EIG curves across criterion settings for a $10 \%, 50 \%$, and $90 \%$ prior probability of guilt, using the same signal detection model as before. Because there is less information to be gained from the witness responses, EIG is lower overall with $10 \%$ or $90 \%$ prior probabilities than with a $50 \%$ prior probability. That is, with a $10 \%$ or $90 \%$ prior probability of guilt, the prior distribution already conveys a lot of information about guilt or innocence. More critically, compared to a 50\% prior probability, the response criterion that maximizes EIG shifts from 0 to 0.31 for a $10 \%$ prior probability and to -0.31 for a $90 \%$ prior probability. These criterion locations are denoted with dashed and dotted vertical lines in Figure 6. Thus, EIG prescribes different procedures for different prior probabilities, but note that, even for the $10 \%$ and $90 \%$ priors, the unbiased criterion value of zero has an EIG value that is very close to EIG at the optimal criterion setting, a point we will elaborate shortly.

These changes in the optimal criterion location are a natural product of the asymmetric uncertainty about guilt introduced by prior information (Wells, Yang, \& Smalarz, 2015). With a $10 \%$ prior probability of guilt, for example, we start with a strong indication that a suspect is innocent. For the $90 \%$ of suspects that are in fact innocent, there is not much room to improve judgment success by reducing uncertainty. That is, learning that an innocent suspect is innocent is not very surprising if one already had information indicating a 90\% chance of innocence, i.e., $\log _{2}(1 / 0.90)=0.15$. For the $10 \%$ of suspects who are actually guilty, however, there is a lot of room for improvement in judgment success. That is, learning that a guilty suspect is guilty is extremely surprising, $\log _{2}(1 / 0.10)=3.32$. This inherent asymmetry prioritizes conservative criteria, which make identifications strong evidence of guilt, over liberal criteria, which make

\footnotetext{
${ }^{7}$ Note that none of our other demonstrations have this property. For the procedural differences explored in those demonstrations, the procedure with the higher EIG is the same for any prior probability of guilt other than 0 or 1 .
} 
rejections strong evidence of innocence. The EIG measure balances the asymmetry in informational value with the frequency of identifications and rejections for each criterion value, revealing the criterion position that optimizes the potential for witness responses to reduce uncertainty about guilt or innocence. Increasing the prior probability of guilt has the opposite effect, and prioritizes procedures with the potential to produce strong evidence for innocence (a liberal criterion, in this example).

The fact that prior information can change the effectiveness of information-gathering procedures is a direct consequence of Bayesian inference and was acknowledged decades ago by eyewitness memory researchers (Wells \& Lindsay, 1980). Nevertheless, some researchers might be troubled by the dependency on prior probability, so we now consider the best response to this dependency.

We are proposing EIG as a research tool to measure the effectiveness of an identification procedure in eyewitness experiments, which tend to have roughly the same number of identification attempts with guilty and innocent suspects (Wells, Yang, \& Smalarz, 2015). In that sense, the dependency on prior probability will rarely be a concern, because the assumed prior probability will be close to the true value for most experiments. The ultimate goal, however, is to learn about procedures that will be effective in real investigations (Clark, 2012). The prior probability of guilt for real identification attempts will vary from case to case and is influenced by a range of factors, such as the other available evidence outside of the identification (Clark, 2012) and the policy for including suspects in an identification procedure (Wells, Yang, \& Smalarz, 2015). In light of the substantial variability in prior probability of guilt across different "real-world" identification attempts, the best policy is to assume a prior probability that is 
maximally robust across different actual prior probabilities, and we will now show that assuming a 50\% prior probability of guilt achieves this desired robustness.

Figure 7 shows the information gain from a simulated show-up procedure for different actual prior probabilities of guilt (different plotting symbols) across a range of criterion values that optimize EIG under different assumed prior probabilities of guilt ( $x$-axis). These results are again based on a signal detection model. Because the effect of prior probability on the optimal criterion value is larger for high $d^{\prime}$, we used a high $d^{\prime}$ value (3). Thus, the robustness illustrated below would be even greater at lower $d^{\prime}$ values.

The circles in Figure 7 show the results with a true prior probability of 0.50 , and the information gain is accordingly highest when the criterion is optimized for an assumed prior probability of 0.50 (which results in an unbiased criterion value; see Figure 6). EIG decreases as the assumed prior probability moves farther below 0.50 (which motivates an increasingly liberal response criterion) or farther above 0.50 (which motivates as increasingly conservative response criterion, see Figure 6).

The open and filled squares show EIG for a true prior probability of 0.25 and 0.75 , respectively. As discussed previously, EIG is lower than for a 50\% prior probability of guilt because with a $25 \%$ or $75 \%$ prior probability of guilt, entropy is relatively low even before a witness response is considered, leaving less room for improvement. The functions are peaked at the true prior probabilities, but the functions are very flat between an assumed prior probability of 0.50 and the true prior probabilities. In other words, using a criterion optimized for a .50 prior probability works just fine for these true prior probabilities, and using a criterion optimized for the true prior probability instead would not provide a meaningful improvement. In fact, the difference in EPG is only .002. 
The open and filled triangles show even more extreme true prior probabilities of guilt of 0.05 and 0.95 , respectively. Information gain is low across the full range for these functions, but this should not be concerning because entropy is already very low in the prior distributions. Again, the functions are peaked at the true prior probabilities, but do not drop much from these values to an assumed prior probability of .50, and the difference in EPG is only .01. Overall, Figure 7 shows that there is not much to be gained by using prior probabilities of guilt other than 0.50 , even if the true prior probability of guilt is far from this value. Moreover, assuming a prior probability far from .50 can be quite detrimental when this assumption is false; for example, the EIG for true base rates of $.25, .5$, and .75 drops steeply as the assumed base rate approached 0 or 1.

An alternative approach would be to try to specify a value - or better yet, a probability distribution - representing the best guess about the prior probability of guilt for real identification attempts, and to seek procedures that optimize information gain under those conditions. For example, we might assume that investigators typically have good reason to believe that a suspect is guilty if they subject that suspect to a lineup, motivating the use of a relatively high prior probability of guilt. To our knowledge, however, there is only one attempt to use data from real investigations to estimate the proportion of guilty suspects. This study was based on a data set for robbery suspects in Houston, and analysis suggested that $35 \%$ of the suspects were actually the culprit (Wixted et al., 2016). This estimate could be used to calculate EIG; however, the prior probability could be different for different precincts, different crimes, and a whole host of other factors (not to mention different estimation procedures, although the method used by Wixted et al. seems to be accurate across a number of data sets with fair lineups, Cohen, Starns, \& Rotello, 2020). Figure 7 shows that calculating EIG with an assumed 50\% 
prior probability of guilt identifies procedures that will be generally effective regardless of the true prior probability of guilt, which eliminates the need to try to anticipate particular prior probabilities.

\section{Summary}

In summary, EIG and diagnosticity ratios support different conclusions. Diagnosticity ratios measure the strength of evidence provided by a particular type of response, such as suspect identifications, but they take no account of how often witnesses make the response and ignore the evidence provided by alternative responses. In contrast, EIG considers all factors relevant to a procedure's evidentiary value, including the information gain associated with every possible witness response and how often these responses are obtained from witnesses. Attempting to combine diagnosticity ratios across different types of responses (e.g., identifications and rejections) does not accurately characterize overall uncertainty about guilt or innocence, because, unlike Shannon surprisal (the basis of EIG), diagnosticity ratios do not measure uncertainty on an additive scale. EIG changes for different prior probabilities of guilt, and sometimes changing prior probability can even switch the relative ranking of different procedures. However, assuming a 50\% prior probability of guilt allows researchers to identify procedures that are either optimal or nearly optimal across the full range of prior probability that might characterize a given investigation.

\section{Demonstration 2: EIG versus Area Under the ROC}

\section{Receiver Operating Characteristics (ROCs)}

Receiver Operating Characteristics (ROCs), in the eyewitness context, show the relationship between the probability of identifying a guilty suspect and the probability of 
identifying an innocent suspect across different levels of response bias, which are typically defined based on confidence-rating data (e.g., Mickes et al., 2012). Recently, Mickes et al. demonstrated how ROCs could be used to compare different eyewitness identification procedures, and the ROC methodology has proven to be a valuable tool for eyewitness researchers that has helped to redefine theoretical debates (Lampinen, 2016; Lampinen et al., 2019; Rotello \& Chen, 2016; Smith et al., 2018; Wixted \& Mickes, 2012; Wixted et al., 2017).

This demonstration focuses on lineup size, a basic choice for any identification procedure. Eyewitness researchers have explored lineup sizes from 1 (a show-up) to 120 or more (e.g., Laughery, Alexander, \& Lane, 1971; Levi, 2006, 2012; Seale-Carlisle et al., 2019). Law enforcement agencies predominantly use lineups that contain between 4 and 9 photos, as well as mugbook searches that could include many more than 9 faces (Police Executive Research Forum, 2013). Both the empirical data and simulation-based research (Rotello \& Chen, 2016) suggest that area under the ROC does not differ meaningfully with lineup size.

We used a signal detection model to vary lineup size while holding all other factors constant. The model simulates a simultaneous lineup in which the witness sees all lineup members at the same time. Witnesses select the lineup member with the highest match strength to their memory for the culprit and respond based on this person alone (Clark, 2003; Duncan, 2006; Macmillan and Creelman, 2005, p. 255-259). Alternative decision processes sometime outperform this sort of "take-the-best" model (Wixted, Vul, Wilson, \& Mickes, 2018), but we used it because it has been explored more extensively and it provides a good description of empirical data (Cohen, Starns, Rotello, \& Cataldo, 2020; Lampinen, 2016; Rotello \& Chen, 2016; Smith, Wells, Lindsay, \& Penrod, 2017; Wixted et al., 2017). Two different lineup sizes were simulated using this model, a 15-person lineup and a 1-person lineup (i.e., a show-up), both 
with a $d^{\prime}$ value of 1.5 . To generate the confidence ratings needed to generate an ROC, we assumed that witnesses made confidence ratings from $0-100$ in steps of 10 (i.e., $0,10,20 \ldots, 90$, 100), which requires 10 response criteria for both identifications and rejections (see the caption of Figure 8 for details).

Figure 8 shows the ROCs for the simulated 15-person and 1-person lineups. The first (left-most) ROC point only “counts” identification responses with a confidence level of 100 as identifications, with the $x$ - and $y$-coordinates determined by the proportion of guilty-suspect and innocent-suspect identification attempts that meet this standard. The second point "counts" identification responses with both confidence 100 and confidence 90 , and so forth until the last point, which includes suspect identifications at all confidence levels.

The 15-person lineup ROC is shorter than the show-up ROC because of a process known as filler siphoning whereby identifications that might have been suspect selections are realized as filler selections instead (Wells, 2001; Wells, Smalarz, \& Smith, 2015). In fact, if innocent suspects are no more likely to match the guilty suspect than the fillers (i.e., if lineups are fair, e.g., Colloff, Wade, \& Strange, 2016), then the maximum identification rate for lineups with innocent suspects is 1/LS where LS is the lineup size (Mickes et al., 2012) ${ }^{8}$. This value for the 15-person lineup is marked by the dashed vertical line in Figure 8. Note that the model used for this demonstration implements the filler siphoning process: When an innocent suspect passes the criterion match strength required to make an identification, there is a chance that one or more of

\footnotetext{
${ }^{8}$ The maximum false identification rate is defined by assuming that witnesses always make a selection; that is, they never make a rejection. The highest observed false identification rate will be lower than the maximum if witnesses sometimes reject the lineup. For example, the maximum possible false identification rate for a show-up is 1 , but the highest observed false identification rate (the right-most ROC point) was much lower than this because more of the innocent-suspect strength distribution fell below the identification criterion.
} 
the fillers will nevertheless exceed the suspect's match strength and prevent a false suspect identification.

The effectiveness of identification procedures is usually measured by the area under the ROC curve (Mickes et al., 2012). For lineup ROCs, it is customary to evaluate the partial area under the ROC up to the maximum false identification rate (Mickes et al., 2012). Figure 8 shows that the ROCs for the 15-person lineup and the show-up overlap almost completely across the range of possible false identification rates for the former. Thus, the partial area under the ROC would be very similar for the two procedures. Rotello and Chen (2016) also demonstrated little or no change in partial area under the ROC for lineups with lengths from 1 to 6 . In other words, an ROC analysis suggests that changing the lineup size over a wide range has no meaningful impact on the effectiveness of the identification procedure when all other factors are held constant.

\section{Lineup vs. Show-up}

We will now consider how lineup size highlights differences between the EIG and ROC measures. We calculated EIG for the model-generated show-up and 15-person lineup data using the equations reported in the EIG and EPG Equations for Eyewitness Researchers section. Note that the set of responses is different than in that earlier section. For the 15-person lineup, the witness can make a suspect identification, a filler identification, or a rejection at one of 11 confidence levels (the show-up has the same responses minus the filler identifications). Despite this change, EIG is calculated using the same steps as described previously.

The procedure for calculating EIG from confidence data for the 15-person lineup is shown in Table 2. For simplicity, this table only shows the steps for a subset of the possible confidence levels. The full version is provided in the Appendix. The signal detection model is 
used to determine the likelihood of each response, $x$, for guilty and innocent suspects $(p(x \mid g)$ and $p(x \mid i))$. From these likelihoods, we compute the overall probability of each response $(p(x)$, Equation 28), the posterior probability of guilt for each response ( $p(g \mid x)$, Equation 39), the entropy for each response $(H(Y \mid x)$, Equation 41), the information gain for each response $(I G(x)$, Equation 42), and, finally, the Expected Information Gain (EIG; Equation 43) by weighting the information gain of each response by the probability of obtaining the response from a witness $(p(x) \times I G(x))$ and summing across responses. For the 15-person lineup, the resulting EIG value is 0.16. Following the same process for the show-up yields an EIG of 0.32, indicating that the show-up procedure has substantially higher evidentiary value compared to the 15-person lineup procedure. Indeed, the Expected Probability Gain (EPG) of witness responses is 32\% for the show-up and only $23 \%$ for the 15 -person line-up, a substantial difference in certainty about guilt or innocence that is not evident in the ROCs, which overlap each other nearly completely (Figure 8).

This example suggests that the EIG measure has the potential to dramatically redefine research questions in the eyewitness memory literature. Reviewing previous identification studies reveals that the field has come to a clear consensus on at least one conclusion: Show-ups are a bad identification procedure. In fact, a recent paper noted that show-ups are "much maligned" in the literature and jovially called them "the bane of all identification procedures" (Wells, Yang, \& Smalarz, 2015, p. 116). By factoring in all relevant aspects of the identification procedure and using an appropriate measure of uncertainty, however, EIG reveals a fairly shocking possibility: Show-ups have more evidentiary value than lineups!

We hasten to add that we are not advocating this as an empirical or practical conclusion. The show-up advantage relies on holding all other factors constant between show-ups and 
lineups, and past results convincingly show that these two procedures differ in a number of ways that affect evidentiary value. First, in some circumstances, show-ups can promote a liberal bias to identify a suspect (Kassin, Ellsworth, \& Smith, 1989), and Figure 4 shows that bias can severely reduce the evidentiary value of witness responses. Second, the opportunity to compare faces in a lineup helps witnesses discriminate guilty and innocent suspects based on their memory for the culprit (Colloff \& Wixted, 2020), and higher witness accuracy increases the evidentiary value of witness responses (as in the hypothetical data set considered in the Information Theory and Identifications section). Thus, although we are not necessarily advocating show-ups over lineups, the comparison here does suggest the intriguing possibility that lineups have an inherent cost that accompanies their benefits. We will now consider the basis of this cost and show how acknowledging it might point the way towards more effective identification procedures.

\section{The Role of Fillers}

Why do show-ups provide better information about guilt than lineups when all other factors are held constant? Show-ups have one very useful property when it comes to judging the guilt or innocence of a suspect based on a witness response: The witness always responds to the suspect. The suspect is the only person considered, and the witness must say whether or not they think the suspect was the culprit and, in some cases, provide a confidence rating. This advantage is lost when fillers are added to the identification procedure. Adding fillers introduces the possibility that a witness will fail to respond to the suspect and instead respond to one of the fillers. In hindsight, it seems obvious that it is difficult to judge the guilt of the suspect based on the witness response if the witness does not respond to the suspect. 
We will illustrate this principle by exploring the distributions of match strength in the signal detection model used for this demonstration across a large range of lineup sizes from 2 to 100. Although the current demonstration assumes a simultaneous lineup, the same problem applies to standard sequential lineups in which lineup members are viewed individually.

As a reminder, the model assumes that witnesses choose the one lineup member that best matches their memory for the culprit and then select a response (and a confidence rating) based on the match strength for this lineup member (Duncan, 2006; Macmillan and Creelman, 2005, p. 255-259; Wixted et al., 2018). Sometimes, the lineup member with the strongest match will be one of the fillers, and this is more likely to happen with larger lineups, which introduce more chances for a filler to randomly provide a good match to the witness' memory for the culprit. For example, with the match-strength distributions for guilty suspects and filler/innocent suspects shown in the top panel of Figure 9, lineup sizes of 2, 10, and 100 produce a 50\%, 90\%, and 99\% probability that a filler will be stronger than an innocent suspect. The corresponding probabilities that a filler will be stronger than a guilty suspect are $8 \%, 33 \%$, and $68 \%$. So, with large lineups there is a high probability that the witness will fail to respond to the suspect because the suspect match is exceeded by a filler match.

This example illustrates that eyewitness researchers should consider an additional metaphor for the role of fillers in a lineup procedure. They function not only as a siphon that reduces the number of false suspect identifications (Wells, 2001; Wells, Smalarz, \& Smith, 2015) but also as a curtain that obscures information about the suspect's guilt or innocence. To appreciate why a larger lineup size degrades the evidentiary value of witness responses, we can look "behind the filler curtain" to evaluate whether the match strength values for suspects who "lose" to fillers contain valuable information about guilt. In a standard eyewitness experiment, 
the investigator does not have access to this information, because, on these trials, the witness responded based on their memory for one of the fillers, not the suspect (in our metaphor, the suspect is behind the filler curtain). If the suspect match distributions convey strong information about guilt on these trials, then the investigator missed out on valuable information.

The bottom panel of Figure 9 shows conditional distributions of match strength for suspects when the strongest match was from a filler (i.e., filler-victory lineups). These distributions are shifted down relative to the distributions across all suspects in the top panel because the conditionalization selects suspects who tend to have relatively low match strengths (making them susceptible to losing to a filler). With a lineup size of 2 , the selection effect is very strong, and so the distributions for guilty and innocent suspects overlap to a high degree. This means that the information obscured by filler victories is of moderate value for discriminating guilty and innocent suspects. In other words, not much information about guilt has been lost behind the filler curtain, and getting a response based uniquely on the suspect's match to the culprit would not dramatically improve judgments of guilt and innocence. As lineup size increases, the selection effect decreases because, with many fillers, the suspect match can be relatively high and still have a good chance of being exceeded by a filler match. This abatement of the selection effect has a larger influence on guilty than innocent suspects, a fact that is easiest to see in comparing lineup sizes 10 and 100. Thus, the conditional strength distributions for lineups with guilty and innocent suspects have less and less overlap as lineup size increases. The dispersed distributions for guilty and innocent suspects for large lineup sizes mean that investigators are missing out on some very valuable information. Overall, Figure 9 shows that using more fillers puts more of the information about guilt behind the filler curtain, a pattern that is not evident in an ROC analysis. 
This demonstration reveals that EIG and area under the ROC can support different conclusions for a key procedural choice in any identification procedure. In the remainder of the section, we expound the differences between EIG and area under the ROC that underlie these sorts of differences in interpretation.

\section{The Fundamental Difference between EIG and ROCs}

The preceding demonstration is one manifestation of a general principle: EIG and area under the ROC measure different constructs. Whereas area under the ROC is designed to measure how effectively witnesses can discriminate guilty and innocent suspects, EIG measures how effectively the witness responses can be used to improve judgments about guilt and innocence (Wells, Yang, \& Smalarz, 2015, highlighted this same distinction in comparing the ROC and Bayesian approaches). Thus, from the perspective of EIG, the ultimate judgments of guilt and innocence are made not by the witnesses themselves, but by an outside agent with access to the witness reports, representing people in the role of, say, jurors, detectives, or judges. The properties measured by the two techniques are closely related, of course, and one can devise many scenarios in which EIG and area under the ROC support the same conclusions about the relative efficacy of identification procedures. However, these measures can also diverge in important respects. For example, as we have already seen, even if we assume that adding more fillers has no effect on the overall distributions of match-to-culprit strength for guilty and innocent suspects, it can still degrade the evidentiary value of witness responses by making it less likely that the witness responds based on the suspect.

A more basic example of the fundamental difference between EIG and area under the ROC is the effect of changes in response tendencies, such as overall conservativeness or the strategy for using a confidence scale. Area under the ROC is insensitive to changes in response 
tendencies; in fact, measuring discriminability independently of response tendencies is the entire point of an ROC analysis (Macmillan \& Creelman, 2005). This ability to independently measure discriminability and response bias is often quite useful for exploring the memory and decision processes that underlie eyewitness identifications. The evidentiary value of witness responses, however, can be dramatically affected by changes in response tendencies (see Wells, Yang, \& Smalarz, 2015, for similar arguments).

To illustrate this point, reconsider the effect of response biases on EIG displayed in Figure 4, Panel E. The property measured by area under the ROC remains constant across all of the widely varying EIG values in this plot. That is, the witness responses were generated from a signal detection model that always had the same distributions representing how well guilty and innocent suspects matched a witness' memory for the culprit. However, even if the witnesses maintain the same potential for discriminating guilty and innocent suspects, their responses lose evidentiary value if they adopt an extremely liberal or extremely conservative response policy, meaning that they basically respond the same way every time, regardless of whether the suspect is guilty or innocent.

Therefore, from the standpoint of policy recommendations, ROCs are not the best tool for identifying the most effective identification procedures; indeed, they are specifically designed to correct for some of the factors affecting evidentiary value (Wells, Yang, \& Smalarz, 2015). Although ROCs will certainly continue to play an important role in clarifying theoretical issues in eyewitness memory, policy recommendations should be based on EIG, as it provides a complete measure of evidentiary value.

Critics of the ROC approach have noted another reason why ROCs may fail to capture the full evidentiary value of an identification procedure; namely, ROCs are not affected by the 
proportion of non-identifications that are filler identifications versus rejections, nor are they affected by the distribution of confidence for either of these response categories (Wells, Smalarz, \& Smith, 2015; Wells, Smith, and Smalarz, 2015; Wells, Yang, \& Smalarz, 2015). This is a minor concern if the different types of non-identification responses have similar distributions for guilty and innocent suspects, but these distributions can be starkly different in some circumstances (Wells \& Olson, 2002; Wells, Yang, \& Smalarz, 2015). EIG considers all responses, and any differences in response patterns between guilty and innocent suspects will increase EIG according to how much these differences improve judgments of guilt or innocence.

\section{Summary}

This demonstration shows that EIG and area under the ROC can support different conclusions about the relative effectiveness of different identification procedures, and using EIG reveals that filler identifications might impair the effectiveness of lineup procedures, especially those with large lineup sizes. This effect of lineup size is not detected by area under the ROC, so it provides a good example of how EIG can inspire new research questions in the eyewitness literature. For example, a corollary of this property is that lineup paradigms in which witnesses always respond to the suspect (e.g., Wilson et al 2019) may be particularly useful.

In summary, area under the ROC and EIG are designed to measure different properties. When they disagree, policy recommendations should be based on EIG, as it is a comprehensive measure of the value of witness responses as a source of evidence about the guilt or innocence of suspects. 


\section{Demonstration 3: EIG versus Deviation from Perfect Performance (DPP)}

Most eyewitness studies that evaluate lineup success with ROCs have used measures based on area under the curve, but ROCs have also been interpreted in terms of the accuracy achieved at individual ROC points. We will focus on one such measure proposed by Smith et al. (2019), Deviation from Perfect Performance (DPP), and use it to further explore the implications of using the EIG measure with confidence-rating data.

In its simplest form, the DPP measure is the conditional error rate for guilty suspects plus the conditional error rate for innocent suspects at a given ROC point. Smith et al. (2019) proposed that an identification procedure can be evaluated in terms of the average or minimum DPP value across all points on the ROC (Smith et al. favored using the minimum over the average). DPP prefers the procedure producing a lower value, as errors are minimized.

For example, consider Figure 10, Panel A. The blue circles show an ROC generated from an SDT model by varying the response criterion (see the figure caption for model details). Here we assume that the criteria are associated with confidence ratings. The red plus symbol shows lineup results generated from the same SDT model, but collapsed across all identification confidence levels and all rejection confidence levels. That is, this model only has a single response criterion, corresponding to the identification-rejection boundary. At this collapsed point, witnesses identified $77.3 \%$ of the guilty suspects and $22.7 \%$ of the innocent suspects

Panel B shows the DPP value for each individual ROC point. For example, the DPP for the central ROC point (red plus) is $.454((1-.773)+.227=.454)$. The horizontal lines mark the minimum DPP and average DPP measures. The lowest DPP value is produced by the procedure that ignores confidence ratings; that is, the model with a single response criterion. If the minimum DPP is used to judge the efficacy of a lineup procedure, then in this example, the 
inclusion of confidence ratings does not improve the evidentiary value of the identification procedure. The average DPP is higher when confidence ratings are considered, indicating worse performance. Thus, if the efficacy of this lineup procedure is judged by the average DPP, including confidence ratings actually decreases the evidentiary value.

EIG provides a starkly different evaluation. The blue circles in Panel C shows the EIG associated with each individual ROC point. The red plus, again, shows the EIG calculated on the data collapsed across confidence ratings. Consistent with the DPP evaluation, the highest individual EIG is for the central point, which, again, corresponds to the procedure that ignores confidence ratings. The horizontal line indicates the EIG value produced by using the full confidence distributions. In contrast to DPP, the EIG value for the full distribution of confidence ratings is higher than the EIG for any of the individual ROC points, even though those ROC points were created from the same confidence data. In fact, using the full confidence scale increased EIG from 0.23 to 0.31 (corresponding to EPG values of $27 \%$ and $31 \%$ ). Table 3 outlines the process of calculating EIG for the full confidence data and for the center ROC point.

Thus, unlike the DPP measures, EIG suggests that obtaining confidence ratings is very helpful for distinguishing guilty and innocent suspects. The primary reason for this difference is that, unlike DPP, the EIG measure does not collapse across different levels of the confidence scale; instead, it evaluates the information gain associated with each confidence level and then averages across levels weighted by their probability. High-confidence responses can be associated with substantial information gain (e.g., Wixted et al., 2016), so segregating them from the lower confidence responses reduces overall uncertainty about guilt, as illustrated in Table 3. 


\section{Summary}

In summary, EIG and DPP will often disagree as measures of the effectiveness of an identification procedure: Whereas DPP collapses the confidence scale to determine the error rate for each individual ROC point, EIG considers the evidentiary value of each separate confidence level and then averages across all identification attempts. This example shows that DPP, and indeed any measure based on individual ROC points, cannot capture the full evidentiary value of confidence rating data. In general, this demonstration suggests that allowing witnesses to provide confidence ratings can meaningfully enhance the evidentiary value of an identification procedure.

\section{Demonstration 4: EIG versus Expected Utility}

\section{Expected Utility}

Several studies have evaluated eyewitness identification procedures in terms of expected utility (Ceci \& Freidman, 2000; Clark, 2012; Lampinen, Smith, \& Wells, 2019; Malpass, 2006; Smith et al., 2019). In this approach, an instrumental value, or utility, is specified for each possible outcome of an identification attempt. The possible outcomes are defined by the factorial combination of whether the suspect was actually guilty or innocent and the possible responses from the witness, e.g., identifying or rejecting the suspect in the simplest case. The utilities represent the societal costs and benefits of identification outcomes, such as the benefit of preventing future crimes by incarcerating guilty suspects or the cost of needlessly robbing innocent suspects of their freedom. These costs and benefits must be quantified to calculate expected utility. For example, Smith et al. (2019) explored a simple utility structure in which correct responses, i.e., identifications of guilty suspects and rejections of innocent suspects, 
gained one unit of instrumental value, $u($ correct $)=1$, and incorrect responses, i.e., identifications of innocent suspects and rejections of guilty suspects, lost one unit of instrumental value, $u($ incorrect $)=-1$.

The success of a particular lineup procedure is evaluated as follows: Determine the probability of each outcome, and then use these probabilities to calculate a weighted average of the utilities. For example, for the 10 -second condition in Table 1, 40\% of suspects were guilty and identified, $p(g \& i d)=0.40,10 \%$ were guilty and rejected, $p(g \& r e j)=0.10,5 \%$ were innocent and identified, $p(i \& i d)=0.05$, and $45 \%$ were innocent and rejected, $p(i \&$ rej $)=0.45$. Thus, with the set of utilities used by Smith et al. (2019), the expected utility is given by

$$
\begin{gathered}
p(\text { g\&id }) u(\text { correct })+p(\text { g\&rej }) u(\text { incorrect })+p(\text { i\&id }) u(\text { incorrect })+p(\text { i\&rej }) u(\text { correct })= \\
0.40 \times 1+0.10 \times-1+0.05 \times-1+0.45 \times 1=0.70
\end{gathered}
$$

Changing the utility of each identification outcome will change the expected utility value, sometimes dramatically so, and can also change the relative ranking of alternative identification procedures. As such, this approach is only useful to the extent that appropriate utility values can be specified.

\section{EIG as an Expected Value}

In comparing EIG and expected utility, it is important to note that they both measure the value of witness responses; the only difference is the definition of "value." EIG defines the value of each identification outcome as the intrinsic evidentiary value of that outcome for judging the guilt or innocence of a suspect instead of the instrumental value defined by the societal consequences of the outcome. The key insight is that we can define the evidentiary value of an outcome as the amount of information that can be gained by that outcome. Other than the switch 
from instrumental value to evidentiary value, expected utility and EIG are identical, as we will now demonstrate.

We have already discussed how information theory provides a method for quantifying the degree of error in judgments of guilt and innocence. Here, we will define the evidentiary value of each identification outcome as the change in judgment success from the prior to posterior distribution of uncertainty about guilt, with judgment success measured in terms of Shannon surprisal. Sticking with the notation in which $Y$ denotes the random variable representing the guilt category across suspects and $X$ denotes the random variable representing the witness responses, the evidentiary value of observing that a particular witness made Response $x$ for a particular suspect who belongs to Guilt Category $y$ is

$$
\begin{gathered}
I G(Y=y \mid X=x)=I(y)-I(y \mid x)= \\
\log _{2}(1 / p(y))-\log _{2}(1 / p(y \mid x))= \\
\log _{2}(p(y \mid x) / p(y)) .
\end{gathered}
$$

Considering guilty suspects in the 10-second condition of the hypothetical show-up experiment, witness identifications bring the probability of the true guilt category from $50 \%$ to $89 \%$, thus the evidentiary value of an identification is

$$
\log _{2}(0.89 / 0.50)=0.83 \text { bits }
$$

For innocent suspects, identifications bring the probability of the true guilt category from $50 \%$ to $11 \%(1.00-0.89=0.11)$, so the evidentiary value of an identification is

$$
\log _{2}(0.11 / 0.50)=-2.17 \text { bits }
$$

The negative evidentiary value signals higher judgment error after than before learning the witness response, as the identification of an innocent suspect provides misleading evidence. 
A rejection of a guilty suspect brings the assessed probability of the true guilt category from $50 \%$ to $18 \%$ for an evidentiary value of

$$
\log _{2}(0.18 / 0.50)=-1.47 \text { bits }
$$

and a rejection of an innocent suspect brings the assessed probability of the true guilt category from $50 \%$ to $82 \%$ for an evidentiary value of

$$
\log _{2}(0.82 / 0.50)=0.71 \text { bits }
$$

To calculate EIG, we take the weighted average of these evidentiary values, just like averaging instrumental values to obtain expected utility:

$$
\begin{gathered}
p(g \& i d) \times \log _{2}(p(g \mid i d) / p(g))+p(g \& r e j) \times \log _{2}(p(g \mid r e j) / p(g))+ \\
p(i \& i d) \times \log _{2}(p(i \mid i d) / p(i))+p(i \& r e j) \times \log _{2}(p(i \mid r e j) / p(i))= \\
0.40 \times 0.83+0.10 \times-1.47+0.05 \times-2.17+0.45 \times 0.71=0.40 .
\end{gathered}
$$

This value matches the expected information gain calculated based on entropy; indeed, what we have presented here is just an alternative formula that highlights the relationship between EIG and expected utility.

This example demonstrates that the question of whether to evaluate an identification procedure based on expected information gain or expected utility comes down to whether we want to define effectiveness in terms of evidentiary value or some instrumental value structure. If identification procedures were tied to a specific, known set of instrumental values, then a focus on expected utility would be justified. Unfortunately, this is not the case. Instead, most statements about instrumental value are vague and based on rough rules of thumb, such as an $18^{\text {th }}$ century legal scholar noting that false convictions should be seen as more than 10 times worse than false acquittals (Blackstone, 1769, cited in Clark, 2012). Moreover, these speculations are not consistent across different authors; for example, some have argued that identifications of 
innocent suspects and rejections of guilty suspects should be considered equally costly, as in the set of utilities used by Smith et al. 2019 discussed above (e.g., Malpass, 2006).

The authors cited in the previous paragraph should be praised for their hedging: Their uncertainty is an honest response to the ambiguity created by the fact that identifications can be used to inform a wide variety of decisions that have a wide range of potential consequences (Clark, 2012). To give a few examples, a detective might need to decide whether or not to end the investigation of a suspect after a failed identification attempt, a judge might need to decide if an identification provides sufficient evidence to grant a search warrant, or a juror might need to decide if an identified suspect should be imprisoned for life. Every different scenario has its own consequences and thus its own set of instrumental values. For example, sentencing an innocent person to life in prison certainly imposes a higher cost than issuing a search warrant for an innocent person. Yet, decisions in all of these situations may rely on the same eyewitness response.

We argue that the proper response to this wide variability in the instrumental value is to find procedures that optimize evidentiary value. Having access to more reliable evidence improves decision making across a wide range of scenarios with different decision consequences.

\section{Summary}

EIG is very similar to expected utility, but, rather than considering the instrumental value of each identification outcome, it considers the evidentiary value of each identification outcome; that is, how much the outcome improves judgments of guilt or innocence. We argue that evidentiary value is the proper focus of eyewitness research. The role of researchers is not to impose a value structure on criminal justice outcomes, but to help officials conduct and interpret 
identifications in a way that produces the best possible information about whether suspects are guilty or innocent (Ramos \& Gonzalez-Rodriguez, 2008; Wixted \& Mickes, 2012). EIG summarizes a given procedure's full evidentiary value, and as such it is an invaluable tool in fulfilling this role.

\section{Demonstration 5: EIG and Witness Efficacy - A Reaction Time Example}

We have shown that EIG has different properties and can support different research conclusions compared to other common measures of the effectiveness of an identification procedure. This section illustrates how EIG can promote new discoveries by allowing researchers to address questions that cannot be effectively answered with existing measures. In particular, we present a theoretical demonstration of how EIG can assess the value of using identification response times (RTs) to predict the accuracy of witness responses.

We chose RT as a convenient example that has a well-known theoretical relationship with accuracy (Ratcliff \& McKoon, 2008), but we stress that these methods could be used for any variable, or combination of variables, that could be used to predict witness accuracy. Such variables include estimator variables (Wells \& Olson, 2003) such as viewing distance, viewing time, level of intoxication, and many others. EIG offers a principled method for assessing which estimator variables are important to consider by quantifying the degree to which an estimator variable improves judgments of guilt and innocence. Moreover, EIG does not require that researchers discretize continuous predictors, in contrast to previous applications of the Bayesian approach (e.g., Chapman \& Cahil, 2012, footnote 4).

For our demonstration, we generated responses from a show-up task using the diffusion model, which is, essentially, a dynamic version of signal detection theory (Ratcliff \& McKoon, 2008; see Figure 11 for model parameters). Figure 11 shows the resulting RT distributions, 
which have the positive skew that is universally observed for empirical RT distributions in standard decision tasks (Wagenmakers, 2009). Another standard pattern is that errors tend to be slower than correct responses (Ratcliff \& McKoon, 2008), and this property is important for the current example because it means that RT can be used to predict the accuracy of a witness response, and in turn, the probability that the suspect is guilty or innocent given the witness response (Smith et al., 2000; Sporer, 1992).

We used a standard logistic regression for this purpose, where the response variable coded the suspect's guilt (1) or innocence (0) and the predictor variable was the $z$-score of the log-transformed RT (we took logs to better approximate a normal distribution and $z$-scores to aid in interpretation of slope coefficients). We ran separate models for identification and rejection responses. The maximum likelihood estimates of the intercept coefficient were 1.87 for identifications and -1.98 for rejections, indicating that the probability of guilt was higher overall for identifications $(0.87)$ than for rejections $(0.12)$. The estimated slope coefficient for identifications was -0.99 , meaning that the predicted probability of guilt was highest for fast identifications and decreased for slower identifications. The slope coefficient for rejections was 0.94, meaning that the predicted probability of guilt was lowest for fast rejections and increased for slower rejections. Overall, these slope coefficients show the desired pattern: Fast responses were more likely to be correct than slow responses, so fast identifications provide particularly strong evidence for guilt and fast rejections provide particularly strong evidence for innocence.

The logistic equations return the predicted probability that the suspect is guilty for every identification attempt in the data set from Witness $w$ based on Response $x_{w}$ and Reaction Time $t_{w}$. To calculate EIG, we used these predictions as the posterior probability of guilt, $p\left(g \mid x_{w} \& t_{w}\right)$, or innocence, $p\left(i \mid x_{w} \& t_{w}\right)$, for each identification attempt, calculated the resulting change in 
Shannon surprisal relative to the appropriate prior (e.g., Equations 42 or 46), and averaged these values across attempts (i.e., across witnesses). We assume here that each witness sees either a single guilty or innocent suspect. Thus, the information gain, IG, or change in Shannon surprisal, is given by

$$
I G\left(x_{w} \& t_{w}\right)=\log _{2}\left(\frac{p\left(g \mid x_{w} \& t_{w}\right)}{p(g)}\right)
$$

for a guilty suspect, and

$$
I G\left(x_{w} \& t_{w}\right)=\log _{2}\left(\frac{p\left(i \mid x_{w} \& t_{w}\right)}{p(i)}\right)
$$

for an innocent suspect. The expected information gain is then calculated as

$$
E I G=\frac{1}{N} \sum_{w} I G\left(x_{w} \& t_{w}\right)
$$

where $N$ is the total number of identification attempts. For the simulated data set, the resulting EIG value is 0.47 , corresponding to an EPG of $38 \%$.

To assess the value of using RT as a predictor of witness accuracy, we compared this value to the EIG value produced by using witness responses without considering RT. This comparison EIG value is 0.37 , corresponding to an EPG of $34 \%$. Thus, using RT as a predictor of witness accuracy improved the evidentiary value of the witness responses. The EIG difference is clearly influenced by the strength of the relationship between the predictor variable and witness accuracy, but it is not just another way of expressing this relationship strength. Instead, the EIG difference combines all relevant factors to measure how much using the predictor variable can reduce uncertainty about guilt, given the witness responses.

Again, although we chose RT as a simple example, the EIG measure can be used for any combination of predictors and any prediction model. Thus, it is a critical tool for investigating estimator variables that can be measured to guide interpretation of witness responses. 


\section{Conceptual Summary of EIG Demonstrations}

We provided five demonstrations of the strengths of using EIG to evaluate the evidentiary value of a lineup procedure.

The first demonstration compared EIG to diagnosticity ratios, which are computed by dividing the likelihoods of observing a particular witness response (typically an identification) for guilty and innocent suspects (Wells \& Lindsay, 1980). We used a signal detection model to determine the effect of varying response bias from very liberal to very conservative in a show-up procedure, representing situations in which witnesses are very willing or very reluctant to identify the suspect, respectively. The diagnosticity ratio for identifications increases to an arbitrarily high value as responding becomes more conservative (as noted by Wixted \& Mickes 2012 and Rotello \& Chen, 2016). Conversely, assuming a 50\% prior probability of guilt, EIG is highest when witnesses are unbiased, that is, when they are equally likely to err by identifying an innocent suspect and by rejecting a guilty suspect. These differences in interpretation arise because, whereas the diagnosticity ratio ignores many of the factors that are relevant to the evidentiary value of an identification procedure, EIG considers all of these factors. Although it is possible to evaluate diagnosticity ratios for rejections as well as identifications (e.g., Wells \& Lindsay, 1980), diagnosticity ratios do not measure uncertainty on an additive scale (Figure 5), and so averaging diagnosticity ratios does not validly index the overall evidentiary value.

The first demonstration also explored the effect of differences in prior probability on EIG. Changing the prior probability of guilt can change the relative EIG values of different procedures. With a low prior probability of guilt, EIG is highest when witnesses respond conservatively, and with a high prior probability, EIG is highest when witnesses respond liberally. We noted that the prior probability of guilt is likely to vary across different 
investigations (Clark, 2012; Wells, Yang, \& Smalarz, 2015). Thus, policy recommendations should prioritize procedures that are generally effective across a broad range of prior probabilities. We showed that optimizing EIG under the assumption of a 50\% prior probability of guilt achieves this desired robustness. That is, even when the true prior probability of guilt is far from $50 \%$, the optimal procedure identified under the assumption of a $50 \%$ prior probability of guilt is nearly as effective as the optimal procedure identified with the true prior probability (Figure 7).

The second demonstration compared EIG and area under the receiver operating characteristic (ROC). ROCs relate the probability of identifying a guilty suspect to the probability of identifying an innocent suspect across different levels of response bias. The area under the ROC is often used to measure the efficacy of a given identification procedure. This demonstration showed that EIG and area under the ROC can support different conclusions about the relative effectiveness of different identification procedures. For example, whereas changing response tendencies, such as shifts in overall response bias, can dramatically reduce EIG, they should have little or no effect on area under the ROC. Moreover, any information about guilt or innocence carried by the different types of non-identification responses (e.g., rejections or filler identifications) will be detected by EIG but will have no effect on area under the ROC.

The second demonstration also considered the effect of adding fillers to a lineup. Using a common signal detection model for lineup tasks (Macmillan and Creelman, 2005, p. 255-259), we showed, surprisingly, that adding fillers to a lineup degrades the evidentiary value of witness responses if all other factors are held constant. In fact, a show-up (i.e., a 1-person lineup) produces the highest EIG value. Area under the ROC gave little to no indication of this drop in evidentiary value. Empirical evidence suggests that many factors vary between lineups and 
show-ups, and many of these changes are advantageous for the lineup procedure (Kassin et al., 1989; Colloff \& Wixted, 2020). Our demonstration suggests that these factors might be hiding an inherent disadvantage of the lineup procedure, that is, the witness does not always provide a response to the suspect. This idea motivates future work on new procedures that maintain the advantages of a lineup while ensuring that the witness always directly responds to the suspect. Although ROCs have proven to be a valuable tool for exploring various research questions in eyewitness research, EIG can add to this tool kit and inspire new research questions.

The third demonstration compared EIG and Deviation from Perfect Performance (DPP), an ROC-based measure that has been proposed as an alternative to area under the ROC (Smith et al., 2019). Each point of a confidence-rating ROC is created by collapsing across a range of confidence levels. DPP defines the effectiveness of an identification procedure based on the error rate at these individual ROC points. We used a signal detection model to determine the effect of either including or ignoring confidence ratings in an identification procedure. Whereas DPP found no benefit of adding confidence ratings, EIG revealed that using a full confidence scale meaningfully increased evidentiary value. Indeed, the evidentiary value produced by using a full confidence scale was higher than the evidentiary value produced by collapsing the confidence scale at any confidence level (Figure 10, Panel C). In other words, collapsing a confidence scale to create an individual ROC point erases much of the information about guilt or innocence carried by the confidence data. This demonstration shows that DPP, and indeed any measure based on individual ROC points, cannot capture the full evidentiary value of confidence rating data.

The fourth demonstration compared EIG and expected utility. In the expected utility approach, an instrumental value, or utility, is assigned to each outcome of an identification 
procedure based on its societal consequences (Ceci \& Freidman, 2000; Clark, 2012; Lampinen, Smith, \& Wells, 2019; Malpass, 2006; Smith et al., 2019). For example, this involves specifying how much is it worth to society to have a witness identify a guilty suspect and how much it costs society to have a witness identify an innocent suspect. Once these instrumental values are assigned, the expected utility is computed by weighing the utility of each individual outcome by the probability of that outcome and summing over outcomes. We used a hypothetical show-up data set (Table 1) to show that expected utility and EIG are identical measures except for of one key difference: EIG replaces the instrumental value of each outcome with its evidentiary value, i.e., how much the outcome improves judgments of guilt or innocence. We note that the instrumental value of identification outcomes is very difficult to specify and can be widely variable across different situations. Thus, we conclude that optimizing evidentiary value is the proper goal for policy recommendations. The role of eyewitness memory researchers is not to impose a value structure on criminal justice outcomes, but to help officials conduct and interpret identifications in a way that produces the best possible information about whether suspects are guilty or innocent (Ramos \& Gonzalez-Rodriguez, 2008; Wixted \& Mickes, 2012). EIG summarizes a given procedure's full evidentiary value, and, as such, it is an invaluable tool in fulfilling this role.

The fifth and final demonstration showed how EIG can be used to assess the value of using predictors of witness accuracy to improve judgments of guilt or innocence. We focused on a particular predictor, response time (RT), i.e., how long it took the witness to make an identification decision. Using the diffusion model (Ratcliff, 1978), we simulated data with a known relationship between RT and accuracy, whereby faster identification decisions were more accurate than slower decisions (Ratcliff \& McKoon, 2008). We used logistic regression to 
predict witness accuracy for identifications and rejections with RT as a continuous predictor. The regression was then used to define the posterior probability of guilt on each identification attempt. The results showed that using RT to predict witness accuracy produced a meaningful increase in EIG. This method could be adapted to any combination of predictors and any prediction model, and indeed, it is a standard metric for evaluating the success of classification algorithms in computer science and engineering (Quinlan, 1986). Because there are a huge range of ecological variables that influence witness accuracy (Wells \& Olson, 2003), exploring the information value of different predictors of witness accuracy is an important direction for eyewitness research. Developing effective methods for predicting witness accuracy could dramatically improve judgments of guilt and innocence, and EIG is the proper tool to guide the search for better prediction methods.

\section{Conclusion}

The principles of probabilistic reasoning (McElreath, 2016) and information theory (Shannon, 1948) provide invaluable quantitative tools for assessing the effectiveness of evidence-gathering practices. We applied these principles to develop a comprehensive method for measuring the evidentiary value of eyewitness identification procedures. We built on previous work that defined the effectiveness of identification procedures based on how much the procedure changed the probability of guilt in a Bayesian analysis (Wells \& Lindsay, 1980). We showed how this approach can be extended to define the Expected Information Gain (EIG) of an identification procedure by measuring uncertainty on the Shannon surprisal scale and averaging the change in uncertainty across a set of identification attempts. This provides a comprehensive measure of a procedure's overall evidentiary value. 
In a series of theoretical demonstrations, we showed that EIG often supports different research conclusions than existing measures of the effectiveness of an identification procedure. In every case, the difference arose because the existing measure overlooked one or more of the factors affecting evidentiary value, whereas EIG incorporates all relevant aspects of identification performance. EIG also has broader applicability than the existing assessment tools, because it can be used to compare any procedures, even when the procedures give witnesses different response options and even when there are tradeoffs in evidentiary value (i.e., one procedure does better in reducing uncertainty for identifications but another does better for rejections). EIG can also be used to evaluate the value of using continuous predictors of witness accuracy, such as response time, allowing researchers to identify important estimator variables using a metric that is targeted to the ultimate goal of identification procedures; namely, reducing uncertainty about guilt or innocence.

Overall, the current work shows that EIG is an important measure for eyewitness memory research, and that it provides a complete assessment of a procedure's potential to inform judgments of guilt or innocence. Thus, EIG supersedes all other measures for research designed to inform policy recommendations for criminal investigations. 


\section{References}

Attneave, F. (1959). Applications of information theory to psychology: A summary of basic concepts, methods, and results. Henry Holt.

Benish, W. A. (1999). Relative entropy as a measure of diagnostic information. Medical Decision Making: an International Journal of the Society for Medical Decision Making, 19, 202-206.

Bickel, J. E. (2007). Some Comparisons among Quadratic, Spherical, and Logarithmic Scoring Rules. Decision Analysis, 4, 49-65.

Blackstone, W. (1769). Commentaries on the Laws of England, Vol. II, Book IV. New York, NY: Duyckinck, Long, Collins \& Hannay, and Collins \& Co.

Brewer, N., Keast, A., \& Rishworth, A. (2002). The confidence-accuracy relationship in eyewitness identification: The effects of reflection and disconfirmation on correlation and calibration. Journal of Experimental Psychology: Applied, 8, 44-56. DOI: 10.1037//1076-898X.8.1.44

Campbell, W.M., Reynolds, D. A., Campbell, J. P., \& Brady, K. J. (2005). Estimating and evaluating confidence for forensic speaker recognition. in Proc. of ICASSP, 2005, pp. $717-720$.

Ceci, S. J., \& Friedman, R. D. (2000). The suggestibility of children: Scientific research and legal implications. Cornell Law Review, 86, 33-108.

Chaitin, G. J. (1975). Randomness and mathematical proof. Scientific American, 232, 47-52.

Chapman, S. D., \& Cahill, B. S. (2012). Witnesses' memory for lineup fillers postdicts their identification accuracy. Journal of Applied Research in Memory and Cognition, 1, 11-17. 
Clark, S. E. (2003). A memory and decision model for eyewitness identification. Applied Cognitive Psychology, 17, 629-654. Doi: 10.1002/acp.891

Clark, S. E. (2012). Costs and benefits of eyewitness identification reform: Psychological science and public policy. Perspectives on Psychological Science, 7, 238-259. doi:10.1177/1745691612439584

Cohen, A. L., Starns, J. J., Rotello, C. M., \& Cataldo, A. M. (2020). Estimating the proportion of guilty suspects and posterior probability of guilt in lineups using signal-detection models. Cognitive Research: Principles and Implications. 5:21 Doi: 10.1186/s41235-020-002194

Colloff, M. F., Wade, K. A., \& Strange, D. (2016). Unfair lineups make witnesses more likely to confuse innocent and guilty suspects. Psychological Science, 27, 1227-1239.

Colloff, M. F., \& Wixted, J. T. (2020). Why are lineups better than showups? A test of the filler siphoning and enhanced discriminability accounts. Journal of Experimental Psychology: Applied, 1, 124-143.

Cover, T. M. \& Thomas, J. A. (2006). Elements of Information Theory, 2nd ed., Wiley Interscience, 2006.

Crupi, V., Nelson, J. D., Meder, B., Cevolani, G., Tentori, K. (2018). Generalized information theory meets human cognition: Introducing a unified framework to model uncertainty and information search. Cognitive Science, 42, 1410-1456.

Cutler, B. L., Penrod, S. D., \& Martens, T. K. (1987). The reliability of eyewitness identification: The role of system and estimator variables. Law and Human Behavior, 11, 233-258.

Dallenbach, K. M. (1913). The relation of memory error to time-interval. Psychological Review, 20, 323-337. 
Deffenbacher, K. (1980). Eyewitness accuracy and confidence: Can we infer anything about their relationship? Law and Human Behavior, 44, 243-260.

Duncan, M. (2006). A signal detection model of compound decision tasks. (Tech Note DRDC TR 2006-256). Toronto: Defence Research and Development Canada.

Fienberg, S. E. (2006). When did Bayesian inference become "Bayesian"? Bayesian Analysis, 1, $1-40$.

Fulero, S. M. (2009). System and estimator variables in eyewitness identification: A review. In D. A. Krauss \& J. D. Lieberman (Eds.), Psychological Expertise in Court: Psychology in the Courtroom (pp. 57-78). Burlington VT: Ashgate Publishing.

Gneiting, T., \& Raftery, A. E. (2007). Stirctly proper scoring rules, prediction, and estimation. Journal of the American Statistical Association, 102, 359-378.

Gong, D., Li, Z., Tao, D., Liu, J., \& Li, X. (2015). A maximum entropy feature descriptor for age invariant face recognition. Proceedings of the IEEE conference on computer vision and pattern recognition, 5289-5297.

Greenspan, R. L., \& Loftus, E. F. (2020). Eyewitness confidence malleability: Misinformation as post-identification feedback. Law and Human Behavior, 4, 194-208. DOI: $10.1037 / \mathrm{lhb} 0000369$

Gronlund, S. D., Carlson, C. A., Dailey, S. B., \& Goodsell, C. A. (2009). Robustness of the sequential lineup advantage. Journal of Experimental Psychology: Applied, 15, 140-152. https://doi.org/10.1037/a0015082.

Gronlund, S. D., Carlson, C. A., Neuschatz, J. S., Goodsell, C. A., Wetmore, S. A., Wooten, A., \& Graham, M. (2012). Showups versus lineups: An evaluation using 
ROC analysis. Journal of Applied Research in Memory and Cognition, 1, 221228. doi:10.1016/j.jarmac.2012.09.003

Juslin, P., Olsson, N., \& Winman, A. (1996). Calibration and diagnosticity of confidence in eyewitness identification: Comments on what can be inferred from the low confidenceaccuracy correlation. Journal of Experimental Psychology: Learning, Memory, and Cognition, 22, 1304-1316.

Kassin, S. M., Ellsworth, P. C., \& Smith, P. L. (1989). The 'general acceptance' of psychological research on eyewitness testing: A survey of the experts. American Psychologist, 44, 10891098.

Lampinen, J. M. (2016). ROC analyses in eyewitness identification research. Journal of Applied Research in Memory and Cognition, 5, 21-33. Doi: 10.1016/j.jarmac.2015.08.006

Lampinen, J. M., Smith, A. M., \& Wells, G. L. (2019). Four utilities in eyewitness identification practice: Dissociations between receiver operating characteristic (ROC) analysis and expected utility Analysis. Law and Human Behavior, 23, 36-44. Doi: 10.1037/lhb0000309

Laughery, K. R., Alexander, J.F., \& Lane, A. B. (1971). Recognition of human faces: Effects of target exposure time, target position, pose position, and type of photograph. Journal of Applied Psychology, 55, 477-483.

Levi, A. M. (2006). An analysis of multiple choices in MSL lineups, and a comparison with simultaneous and sequential ones. Psychology, Crime \& Law, 12, 273-285. Doi: $10.1080 / 10683160500238782$

Levi, A. M. (2012) Much better than the sequential lineup: A 120-person lineup. Psychology, Crime \& Law, 18, 631-640. Doi: 10.1080/1068316X.2010.526120 
Lindsay, R. C. L., Semmler, C., Weber, N., Brewer, N., \& Lindsay, M. R. (2008). How variations in distance affect eyewitness reports and identification accuracy. Law and Human Behavior, 32, 526-535. DOI: 10.1007/s10979-008-9128-х

Loftus, G. R. (1985). Picture perception: Effects of luminance on available information and information-extraction rate. Journal of Experimental Psychology: General, 114, 342356.

Macmillan, N. A., \& Creelman, C. D. (2005). Detection Theory: A User's Guide (2nd ed.). Mahwah, NJ: Lawrence Erlbaum Associates.

Malpass, R. S. (2006). A policy evaluation of simultaneous and sequential lineups. Psychology, Public Policy, and Law, 12, 394 - 418. Doi: 10.1037/1076-8971.12.4.394

Malpass, R. S., \& Kravitz, J. (1969). Recognition for faces of own and other race. Journal of Personality and Social Psychology, 13, 330-334.

McElreath, R. (2016). Statistical Rethinking: A Bayesian Course with Examples in R and STAN. Boca Raton: CRC Press/Taylor \& Francis Group.

McGrayne, S. B. (2011). The Theory that Would Not Die. New Haven, CT: Yale University Press.

Memon, A., Hope, L., \& Bull, R. H. C. (2003). Exposure duration: Effects on eyewitness accuracy and confidence. British Journal of Psychology, 94, 339-354.

Mickes, L., Flowe, H. D., \& Wixted, J. T. (2012). Receiver operating characteristic analysis of eyewitness memory: Comparing the diagnostic accuracy of simultaneous and sequential lineups. Journal of Experimental Psychology: Applied, 18, 361-376.

Mickes, L., Seale-Carlisle, T. M., Wetmore, S. A., Gronlund, S. D., Clark, S. E., Carlson, C. A., ... Wixted, J. T. (2017). ROCs in eyewitness identification: Instructions versus 
confidence. Applied Cognitive Psychology, 31, 467-477. https://doi.org/ 10.1002/acp.3344.

National Institute of Justice (1999). Eyewitness evidence: A guide for law enforcement. Washington DC: US Department of Justice

National Research Council (2014). Identifying the culprit: Assessing eyewitness identification. Washington, DC: National Academies Press.

Osterburg, James \& Parthasarathy, T. \& Raghavan, T. (1977). Development of a Mathematical Formula for the Calculation of Fingerprint Probabilities Based on Individual Characteristics. Journal of The American Statistical Association, 72. 772-778. 10.1080/01621459.1977.10479954.

Police Executive Research Forum (2013). A National Survey of Eyewitness Identification Procedures in Law Enforcement Agencies. Washington, DC: US Department of Justice

Price, H. L., \& Fitzgerald, R. J. (2016). Face-off: A new identification procedure for child eyewitnesses. Journal of Experimental Psychology: Applied, 22, 366-380.

Quinlan, J. R. (1986). Induction of decision trees. Machine Learning, 1, 81-106.

Ramos, D. \& J. Gonzalez-Rodriguez, J. (2008). Cross-entropy Analysis of the Information in Forensic Speaker Recognition. Proceedings of IEEE Odyssey, Odyssey 2008: The Speaker and Language Recognition Workshop.

Ramos, D., Gonzalez-Rodriguez, J., Zadora, G., Zieba-Palus, J., \& Aitken, C. (2007). Information-theoretical comparison of likelihood ratio methods of forensic evidence evaluation. Third International Symposium on Information Assurance and Security, 411416. 
Ratcliff, R. (1978). A theory of memory retrieval. Psychological Review, 85, 59-108.

Ratcliff, R., \& McKoon, G. (2008). The diffusion decision model: Theory and data for twochoice decision tasks. Neural Computation, 20, 873-922.

Robert, C. P. (2007). The Bayesian Choice: From Decision-Theoretic Foundations $t$ Computational Implementation. Springer.

Rotello, C. M., \& Chen, T. (2016). ROC curve analyses of eyewitness identification decisions: An analysis of the recent debate. Cognitive Research: Principles and Implications, 1:10. DOI 10.1186/s41235-016-0006-7

Savage, L. J. (1971). Elicitation of Personal Probabilities and Expectations. Journal of the American Statistical Association, 66, 783-801.

Schklar, J., \& Diamond, S. S. (1999). Juror reactions to DNA evidence: Errors and expectancies. Law and Human Behavior, 23, 159-184.

Schooler, J. W., \& Engstler-Schooler, T. Y. (1990). Verbal overshadowing of visual memories: Some things are better left unsaid. Cognitive Psychology, 22, 36-71. doi:10.1016/00100285(90)90003-m.

Seale-Carlisle, T. M., Wetmore, S. A., Flowe, H. D., \& Mickes, L. (2019). Designing police lineups to maximize memory performance. Journal of Experimental Psychology: Applied, 25, 410-430. DOI: 10.1037/xap0000222

Shannon, C.E. (1948) A Mathematical Theory of Communication. Bell System Technical Journal, $27,379-423,623-656$.

Shapiro, P. N., \& Penrod, S. (1986). Meta-analysis of facial identification studies. Psychological Bulletin, 100, 139-156. 
Smith, A. M., Lampinen, J. M., Wells, G. L., Smalarz, L., \& Mackovichova, S. (2019). Deviation from perfect performance measures the diagnostic utility of eyewitness lineups but partial area under the ROC curve does not. Journal of Applied Research in Memory and Cognition, 8, 50-59. DOI: 10.1016/j.jarmac.2018.09.003

Smith, S. M., Lindsay, R. C. L., \& Pryke, S. (2000). Postdictors of eyewitness errors: Can false identifications be diagnosed? Journal of Experimental Psychology: Applied, 85, 542-550. Doi: 10.1037//0021-9010.85.4.542

Smith, A. M., Wells, G. L., Lindsay, R. C. L., \& Penrod, S. D. (2017). Fair lineups are better than biased lineups and showups, but not because they increase underlying discriminability. Law and Human Behavior, 41, 127-145.

Smith, A. M., Wells, G. L., Smalarz, L., \& Lampinen, J. M. (2018). Increasing the similarity of lineup fillers to the suspect improves the applied value of lineups without improving memory performance: Commentary on Colloff, Wade, and Strange (2016). Psychological Science, 29, 1548-1551.

Sporer, S. L. (1993). Eyewitness identification accuracy, confidence, and decision times in simultaneous and sequential lineups. Journal of Applied Psychology, 78, 22-33.

Steblay, N. M. (1997). Social influence in eyewitness recall: A meta-analytic review of lineup instruction effects. Law and Human Behavior, 21, 283-297.

Wagenmakers, E.-J. (2009). Methodological and empirical developments for the Ratcliff diffusion model of response times and accuracy. European Journal of Cognitive Psychology, 21, 641-671.

Wells, G. L. (1978). Applied eyewitness-testimony research: System variables and estimator variables. Journal of Personality and Social Psychology, 36, 1546. 
Wells, G. L. (2001). Police lineups: Data, theory, and policy. Psychology, Public Policy, and Law, 7, 791-801.

Wells, G. L., \& Lindsay, R. C. L. (1980). On estimating the diagnosticity of eyewitness nonidentifications. Psychological Bulletin, 88, 776-784. doi:10.1037/0033-2909.88.3.776

Wells, G. L., \& Olson, E. A. (2002). Eyewitness identification: Information gain from incriminating and exonerating behaviors. Journal of Experimental Psychology: Applied, 8, 155-167. Doi: 10.1037//1076-898X.8.3.155

Wells, G. L., \& Olson, E. A. (2003). Eyewitness testimony. Annual Review of Psychology, 54, 277-295.

Wells, G. L., Smalarz, L., \& Smith, A. M. (2015). ROC analysis of lineups does not measure underlying discriminability and has limited value. Journal of Applied Research in Memory and Cognition, 4, 313-317. Doi: 10.1016/j.jarmac.2015.08.008

Wells, G. L., Smith, A. M., \& Smalarz, L. (2015). ROC analysis of lineups obscures information that is critical for both theoretical understanding and applied purposes. Journal of Applied Research in Memory and Cognition, 4, 324-328. Doi: 10.1016/j.jarmac.2015.08.010

Wells, G. L., \& Turtle, J. W. (1986). Eyewitness identification: The importance of lineup models. Psychological Bulletin, 99, 320-329.

Wells, G. L., Yang, Y., \& Smalarz, L. (2015a). Eyewitness identification: Bayesian information gain, base-rate effect-equivalency curves, and reasonable suspicion. Law and Human Behavior, 39, 99-122. doi:10.1037//hhb0000125

Wetmore, S. A., Neuschatz, J. S., Gronlund, S. D., Wooten, A., Goodsell, C. A., \& Carlson, C. A. (2015). Effect of retention interval on showup and lineup performance. Journal of Applied Research in Memory and Cognition, 4, 8-14. doi:10.1016/j.jarmac.2014.07.003 
Wilson, B. M., Donnelly, K., Christenfeld, N., \& Wixted, J. T. (2019). Making sense of sequential lineups: An experimental and theoretical analysis of position effects. Journal of Memory and Language, 104, 108-125.

Winkler, R. L. (1969). Scoring rules and the evaluation of probability assessors. Journal of the American Statistical Association, 64, 1073-1078.

Wixted, J. T. (2020). The forgotten history of signal detection theory. Journal of Experimental Psychology: Learning, Memory, and Cognition, 46, 201-233.

Wixted, J. T., \& Mickes, L. (2012). The field of eyewitness memory should abandon "probative value" and embrace Receiver Operating Characteristic analysis. Perspectives on Psychological Science, 7, 275-278.

Wixted, J. T., Mickes, L., Dunn, J. C., Clark, S. E., \& Wells, W. (2016). Estimating the reliability of eyewitness identifications from police lineups. Proceedings of the National Academy of Science, 113, 304-309. doi:10.1073/pnas.1516814112

Wixted, J. T., Mickes, L., Wetmore, S. A., Gronlund, S. D., \& Neuschatz, J. S. (2017). ROC Analysis in Theory and Practice. Journal of Applied Research in Memory and Cognition, $6,343-351$.

Wixted, J. T., Vul, E., Mickes, L., \& Wilson, B. M. (2018). Models of lineup memory. Cognitive Psychology, 105, 81-114. https://doi.org/10.1016/j.cogpsych.2018.06.001.

Zhou, X., Cheng, M., He, X., \& Matsumodo, T. (2014). Exact and approximated outage probability analyses for decode-and-forward relaying system allowing intra-link errors. IEEE Transactions on Wireless Communications, 137062-7071. 10.1109/TWC.2014.2354337 
Table 1.

Response frequencies and probabilities for the hypothetical show-up experiment

\begin{tabular}{|c|c|c|c|c|c|c|}
\hline \multirow[b]{3}{*}{ Guilt Category } & \multicolumn{6}{|c|}{ Witness Response } \\
\hline & \multicolumn{3}{|c|}{ Frequencies } & \multicolumn{3}{|c|}{ Probabilities } \\
\hline & Id. & Rej. & Total & Id. & Rej. & Total \\
\hline & \multicolumn{6}{|c|}{ 10-second Condition } \\
\hline Guilty & 400 & 100 & 500 & .40 & .10 & .50 \\
\hline Innocent & 50 & 450 & 500 & .05 & .45 & .50 \\
\hline \multirow[t]{2}{*}{ Total } & 450 & 550 & 1000 & .45 & .55 & 1 \\
\hline & \multicolumn{6}{|c|}{ 30-second Condition } \\
\hline Guilty & 450 & 50 & 500 & .45 & .05 & .50 \\
\hline Innocent & 25 & 475 & 500 & .025 & .475 & .50 \\
\hline Total & 475 & 525 & 1000 & .475 & .525 & 1 \\
\hline
\end{tabular}

Note. Id. = identification; Rej. = rejection 
Table 2.

Steps in calculating EIG for the 15-person lineup in Demonstration 2

\begin{tabular}{lccccccc}
\hline & $\boldsymbol{p}(\boldsymbol{x} \mid \boldsymbol{g})$ & $\boldsymbol{p}(\boldsymbol{x} \mid \boldsymbol{i})$ & $\boldsymbol{p}(\boldsymbol{x})$ & $\boldsymbol{p}(\boldsymbol{g} \mid \boldsymbol{x})$ & $\boldsymbol{H}(\boldsymbol{Y} \mid \boldsymbol{x})$ & $\boldsymbol{I G}(\boldsymbol{x})$ & $\boldsymbol{p}(\boldsymbol{x}) \times \boldsymbol{I G}(\boldsymbol{x})$ \\
\hline & & & & & & & \\
Response (x) & 0.066 & 0.001 & 0.0335 & 0.9851 & 0.1119 & 0.8881 & 0.02980 \\
$\vdots$ & $\vdots$ & $\vdots$ & $\vdots$ & $\vdots$ & $\vdots$ & $\vdots$ & $\vdots$ \\
Suspect Id. 100 & 0.010 & 0.006 & 0.0080 & 0.6250 & 0.9544 & 0.0456 & 0.00040 \\
Filler Id. 100 & 0.018 & 0.019 & 0.0185 & 0.4865 & 0.9995 & 0.0005 & 0.00001 \\
$\vdots$ & $\vdots$ & $\vdots$ & $\vdots$ & $\vdots$ & $\vdots$ & $\vdots$ & $\vdots$ \\
Filler Id. 0 & 0.032 & 0.079 & 0.0555 & 0.2883 & 0.8665 & 0.1335 & 0.0074 \\
Rej. 100 & 0.000 & 0.000 & 0.0000 & -------- & ------- & -------- & ------- \\
$\vdots$ & $\vdots$ & $\vdots$ & $\vdots$ & $\vdots$ & $\vdots$ & $\vdots$ & $\vdots$ \\
Rej. 0 & 0.019 & 0.047 & 0.0330 & 0.2879 & 0.8660 & 0.1340 & 0.0044 \\
& & & & & & & \\
& & & & & $\boldsymbol{\Sigma} \boldsymbol{p}(\boldsymbol{x}) \times \boldsymbol{I G}(\boldsymbol{x})=\boldsymbol{E I G}=\mathbf{0 . 1 6}$ \\
\hline
\end{tabular}

Notes. Rows for intermediate confidence levels were removed for simplicity; see the Appendix for the full table with all confidence levels. Model predictions were rounded to the nearest $1000^{\text {th }}$, resulting in zero probability for some responses. Zero-probability responses are ignored in the EIG calculations. $p(x \mid g)=$ probability of Response $x$ on identification attempts with guilty suspects; $p(x \mid i)=$ probability of Response $x$ on identification attempts with innocent suspects; $p(x)=$ overall probability of Response $x ; p(g \mid x)=$ posterior probability of guilt for lineup Response $x$ (posterior probability of innocence is $1-p(g \mid x)$ ); H(Y|x)= entropy for Response $x$; $I G(x)$ information gain for Response $x$; EIG = expected information gain. 
Table 3.

Steps for calculating EIG for the show-up in Demonstration 3 with the full confidence scale and with the scale collapsed to create a single ROC point

$\begin{array}{llllllll}\text { Response }(x) & p(x \mid g) & p(x \mid i) & p(x) & p(g \mid x) & H(Y \mid x) & I G(x) & p(\mathbf{x}) \times I G(x)\end{array}$

Full Confidence Data

\begin{tabular}{|c|c|c|c|c|c|c|c|}
\hline Suspect Id. 4 & 0.382 & 0.036 & 0.2090 & 0.9139 & 0.4234 & 0.5766 & 0.1205 \\
\hline Suspect Id. 3 & 0.138 & 0.038 & 0.0880 & 0.7841 & 0.7526 & 0.2474 & 0.0218 \\
\hline Suspect Id. 2 & 0.135 & 0.062 & 0.0985 & 0.6853 & 0.8985 & 0.1015 & 0.0100 \\
\hline Suspect Id. 1 & 0.118 & 0.091 & 0.1045 & 0.5646 & 0.9879 & 0.0121 & 0.0013 \\
\hline Rej. 4 & 0.036 & 0.382 & 0.2090 & 0.0861 & 0.4234 & 0.5766 & 0.1205 \\
\hline Rej. 3 & 0.038 & 0.138 & 0.0880 & 0.2159 & 0.7526 & 0.2474 & 0.0218 \\
\hline Rej. 2 & 0.062 & 0.135 & 0.0985 & 0.3147 & 0.8985 & 0.1015 & 0.0100 \\
\hline Rej. 1 & 0.091 & 0.118 & 0.1045 & 0.4354 & 0.9879 & 0.0121 & 0.0013 \\
\hline \multirow{2}{*}{\multicolumn{8}{|c|}{$\Sigma p(x) \times I G(x)=E I G=0.31$}} \\
\hline & & & & & & & \\
\hline \multirow{2}{*}{$\begin{array}{l}\text { Suspect Id. 1-4 } \\
\text { Rej. 1-4 }\end{array}$} & 0.773 & 0.227 & 0.500 & 0.773 & 0.7727 & 0.2273 & 0.1136 \\
\hline & 0.227 & 0.773 & 0.500 & 0.227 & 0.7727 & 0.2273 & 0.1136 \\
\hline
\end{tabular}

Notes. Model predictions were rounded to the nearest $1000^{\text {th }} \cdot p(x \mid g)=$ probability of Response $x$ on identification attempts with guilty suspects; $p(x \mid i)=$ probability of Response $x$ on identification attempts with innocent suspects; $p(x)=$ overall probability of Response $x ; p(g \mid x)=$ posterior probability of guilt for lineup Response $x$ (posterior probability of innocence is 1 $p(g \mid x)) ; H(Y \mid x)=$ entropy for Response $x ; I G(x)$ information gain for Response $x$; EIG = expected information gain. 

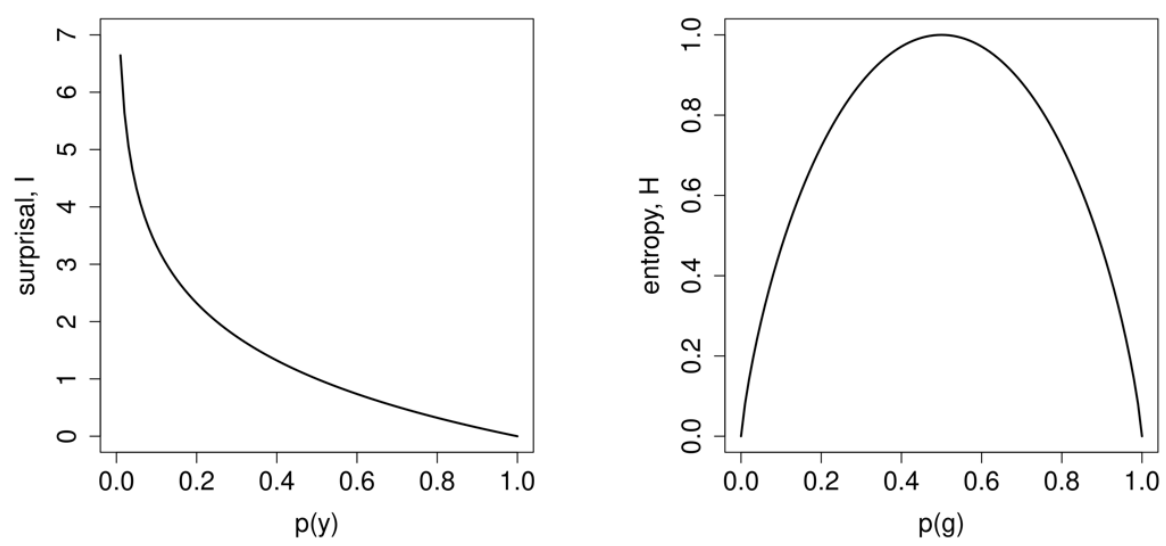

Figure 1.

Relationship of Shannon surprisal and entropy to probability. In the left panel, $p(y)$ denotes the judged probability of an outcome, and $I$ denotes the degree of judgment error as measured by surprisal, or equivalently, the amount on information gained by observing the outcome. In the right panel, $p(g)$ denotes the probability of guilt for a Bernoulli distribution defining uncertainty about guilt or innocence, and $H$ denotes the entropy of the distribution (i.e., the average Shannon surprisal). 

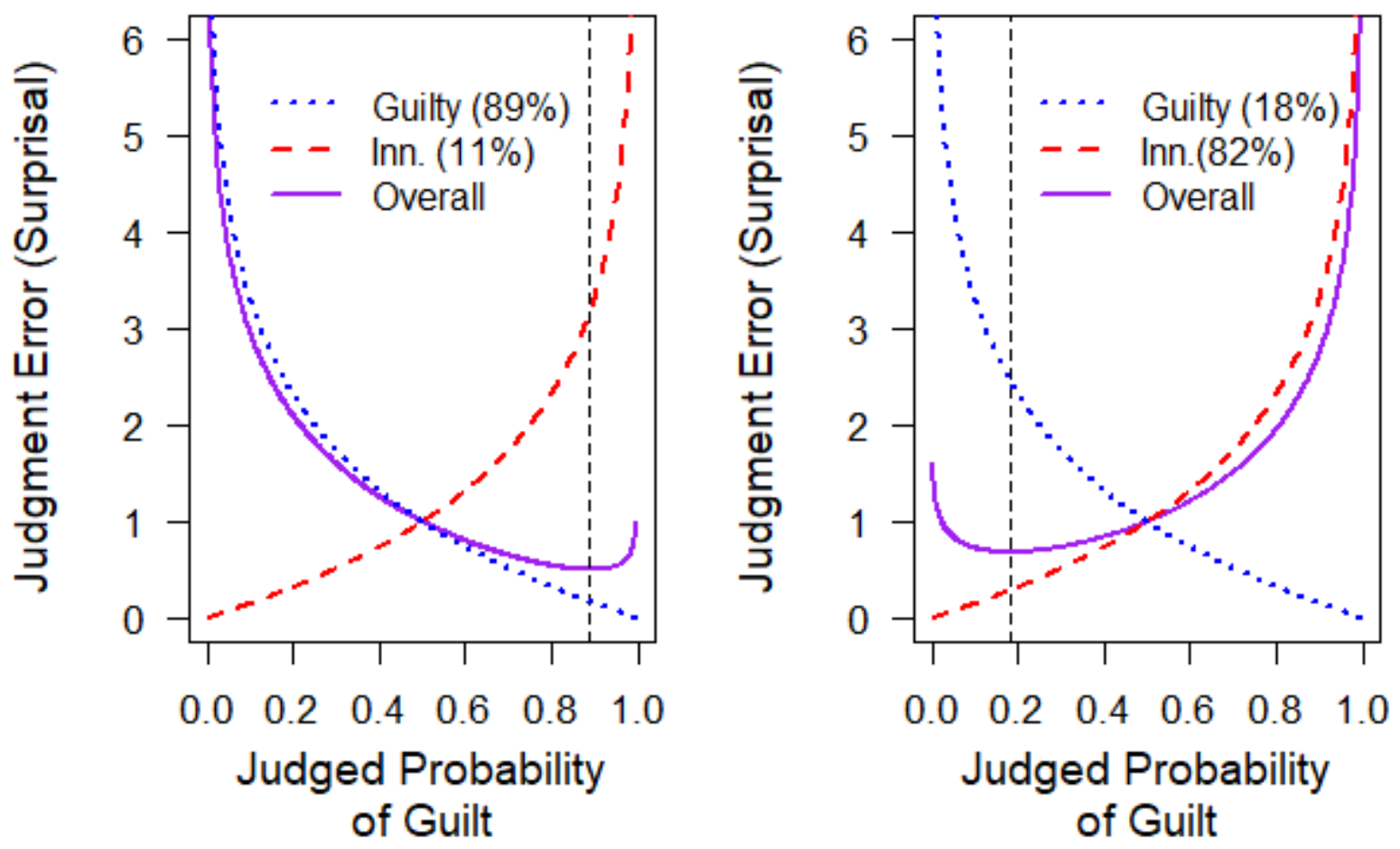

Figure 2.

Judgment error for show-ups that led to identifications (left panel) and rejections (right panel) in the 10-second condition of the hypothetical show-up data set from Table 1. For show-ups with identifications, $89 \%$ had a guilty suspect and $11 \%$ had an innocent suspect. For show-ups with rejections, $18 \%$ had a guilty suspect and $82 \%$ had an innocent suspect. 


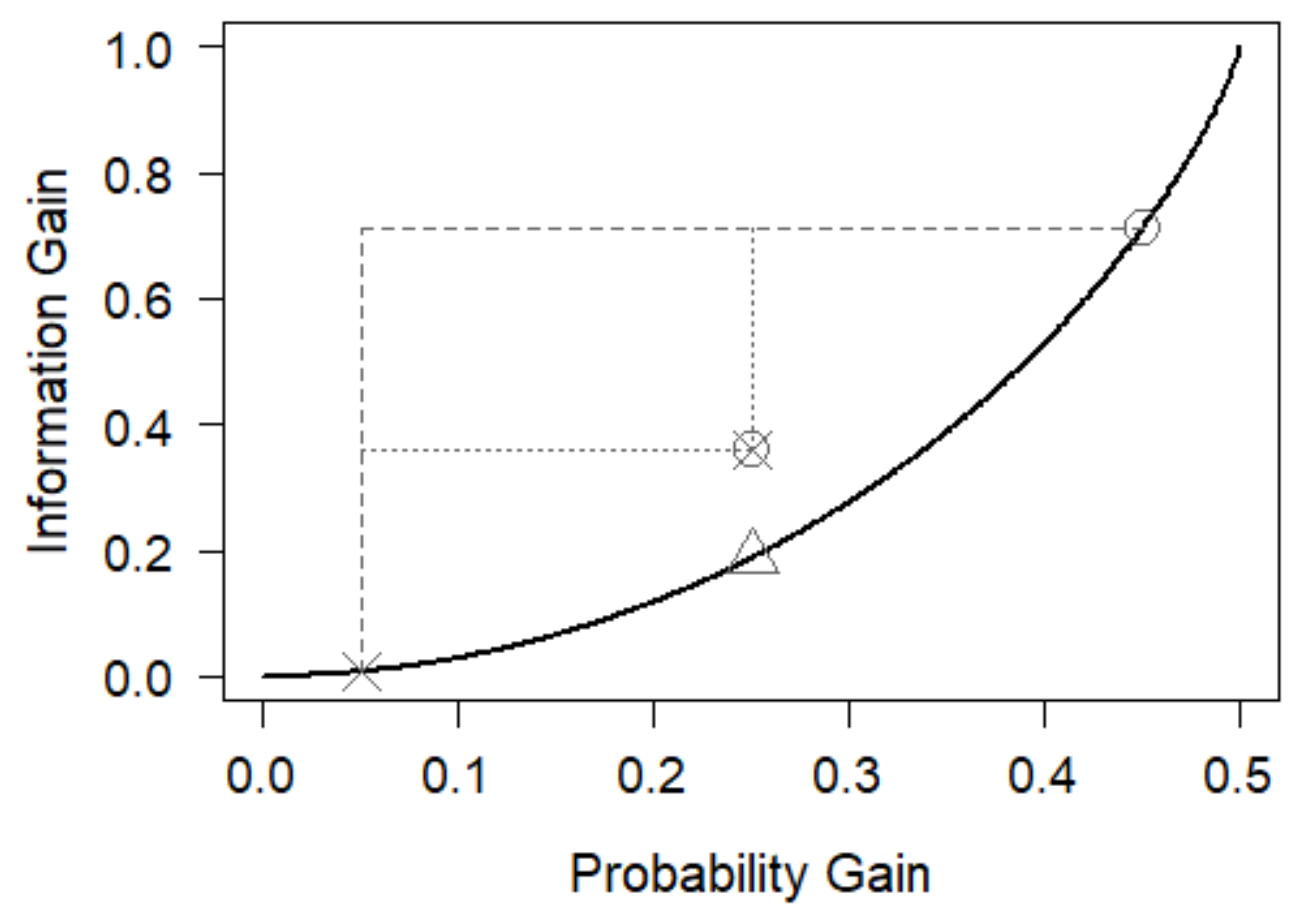

Figure 3.

Relationship between probability gain and information gain when the prior probability of guilt is $50 \%$. The gray points and lines illustrate averaging artifacts on the probability scale. The $\mathrm{x}$, triangle, and circle mark values for a posterior distribution with $55 \%, 75 \%$, and $95 \%$ certainty about guilt category. Averaging across the $55 \%$ and $95 \%$ posteriors produces a different result on the two scales, as shown by the lines and the combined circle-x point. The probability gain average is distorted because this measure is not additive over uncertainty. 

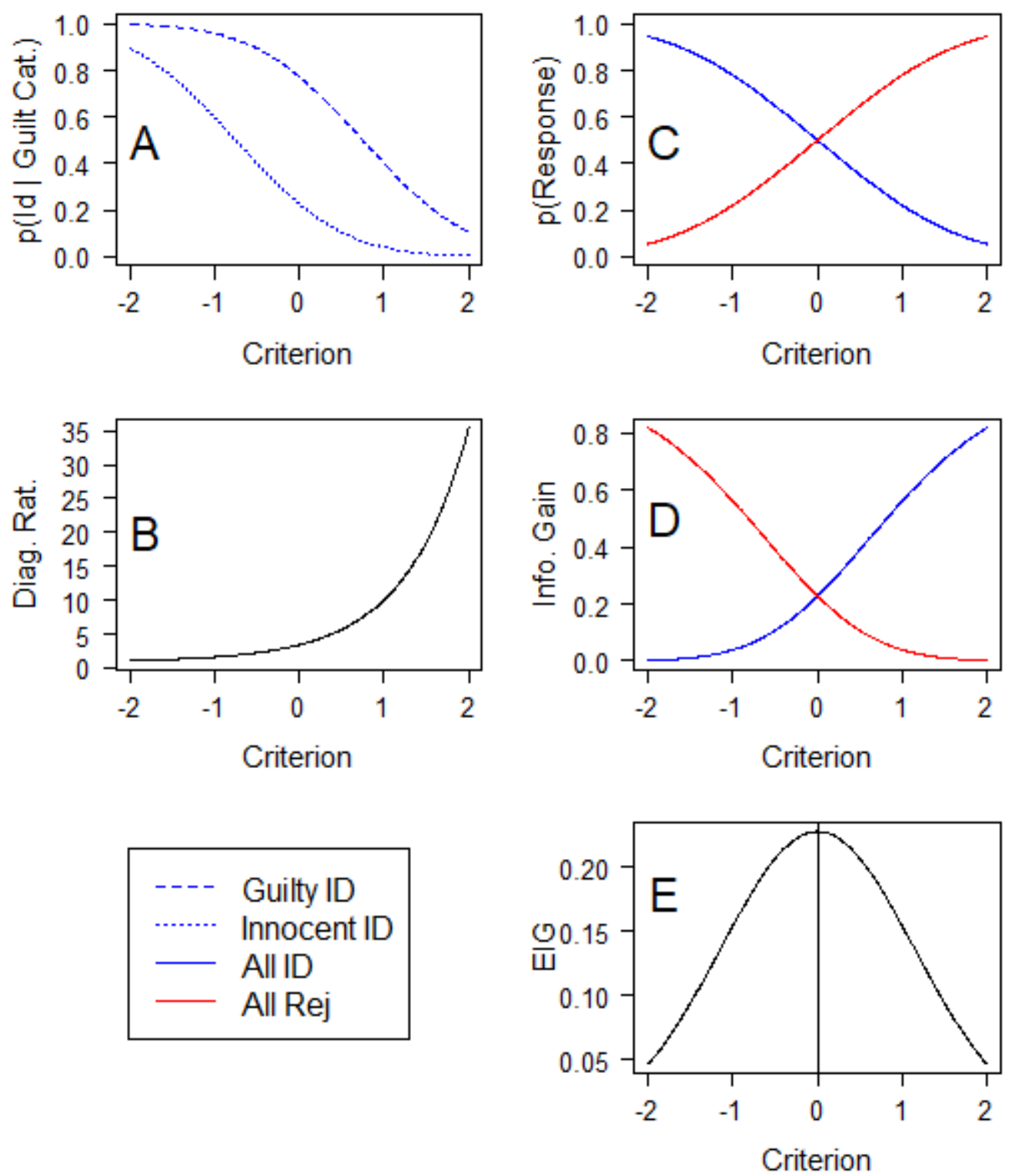

Figure 4.

Illustration of the differences between EIG and diagnosticity ratios for the effect of changing the response criterion in a signal detection model, simulating differences in witnesses' overall willingness to identify a suspect. The model used Gaussian distributions with standard deviations of 1 and means of -0.75 and 0.75 for identification attempts with innocent and guilty suspects, respectively. Thus, $d^{\prime}$ was 1.5 . ID = identification; $R e j=$ rejection . 

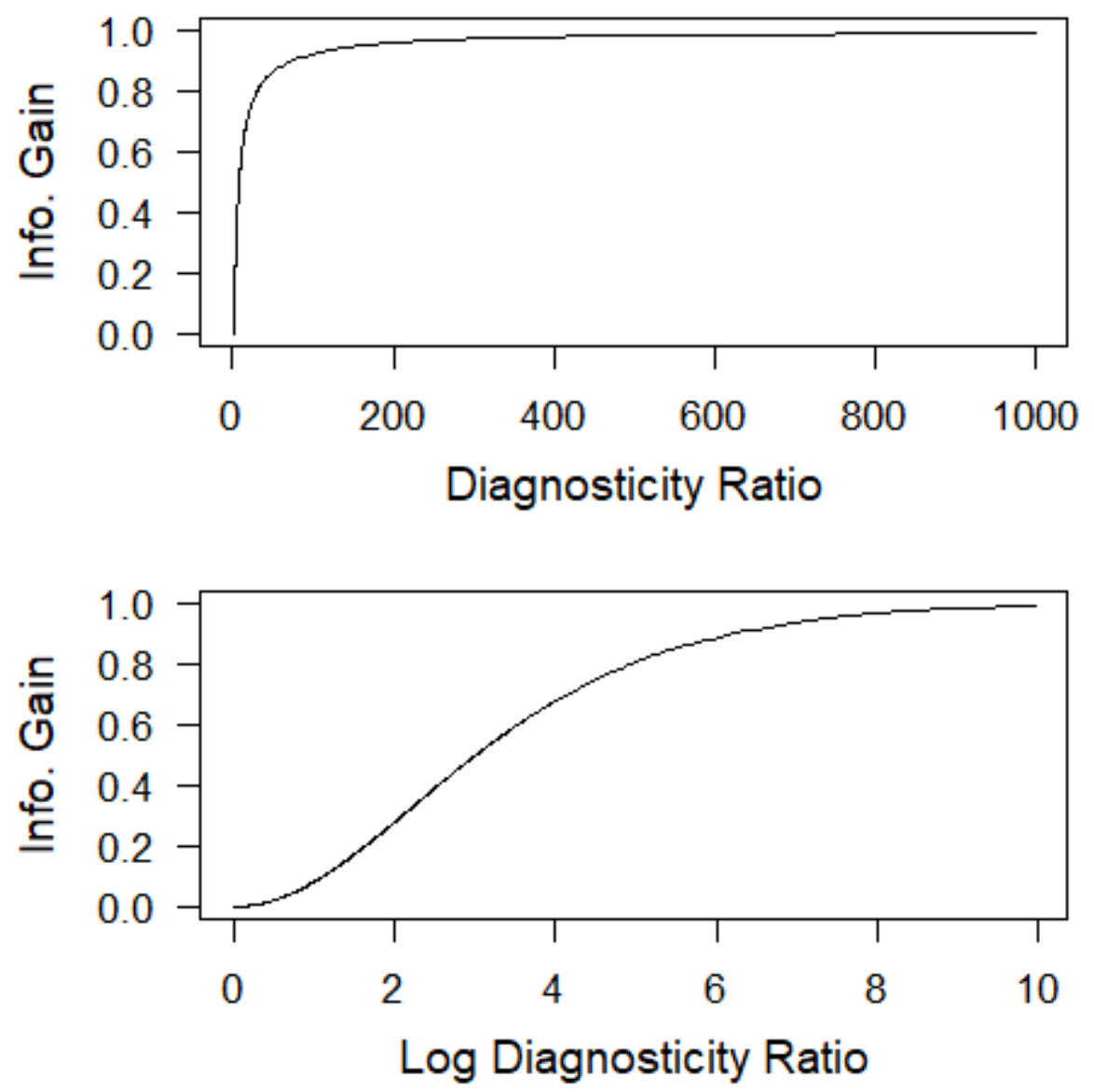

Figure 5.

Relationship between information gain and the diagnosticity ratio (and log diagnosticity ratio) when the prior probability of guilt is $50 \%$. 


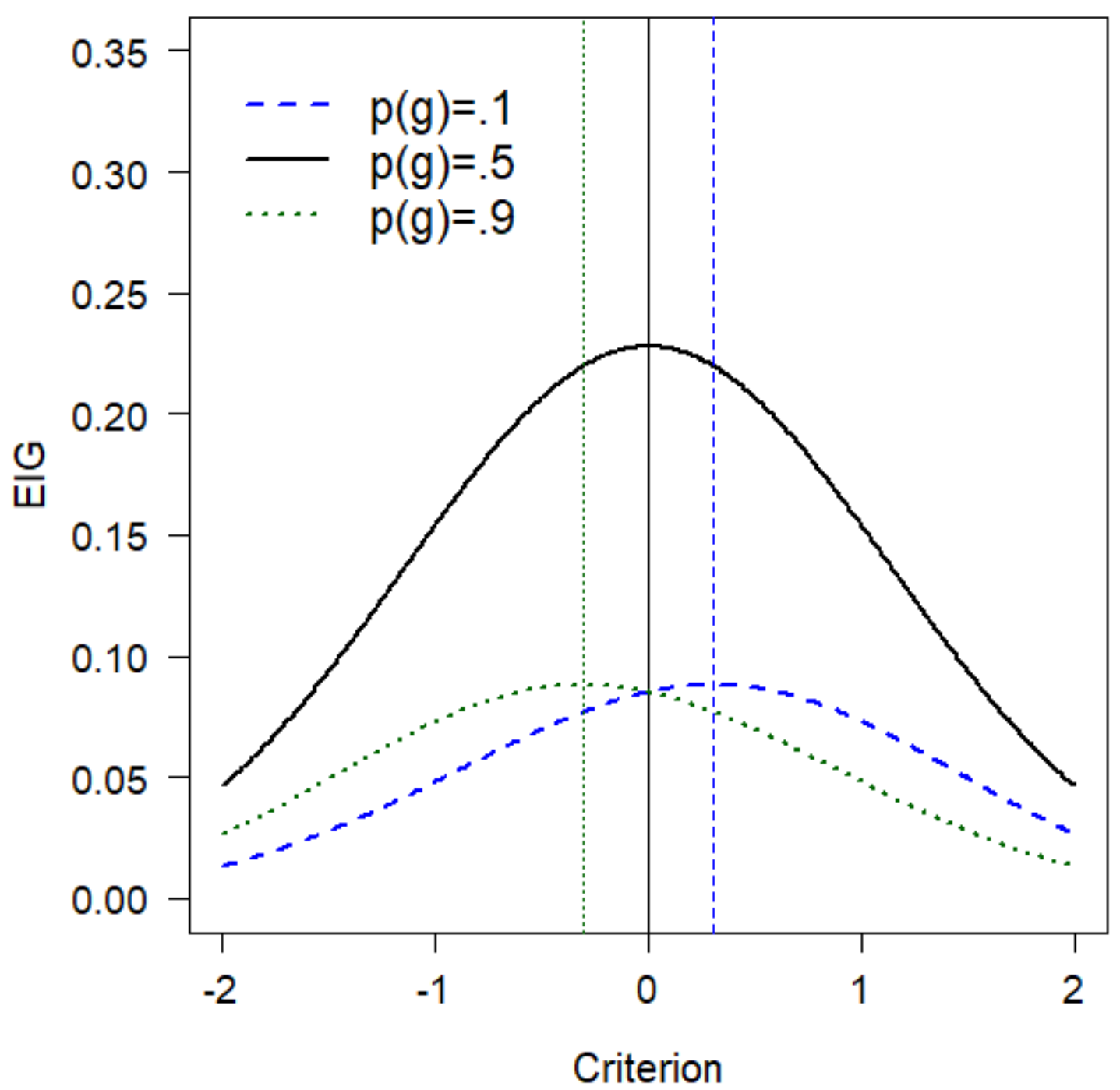

Figure 6.

EIG across different criteria in a signal detection model, simulating differences in witnesses' overall willingness to identify a suspect. $p(g)$ is the prior probability of guilt. The dashed lines mark the criterion position that optimizes the evidentiary value of the witness responses. The model used Gaussian distributions with standard deviations of 1 and means of -0.75 and 0.75 for identification attempts with innocent and guilty suspects, respectively. Thus, $d^{\prime}$ was 1.5 . 


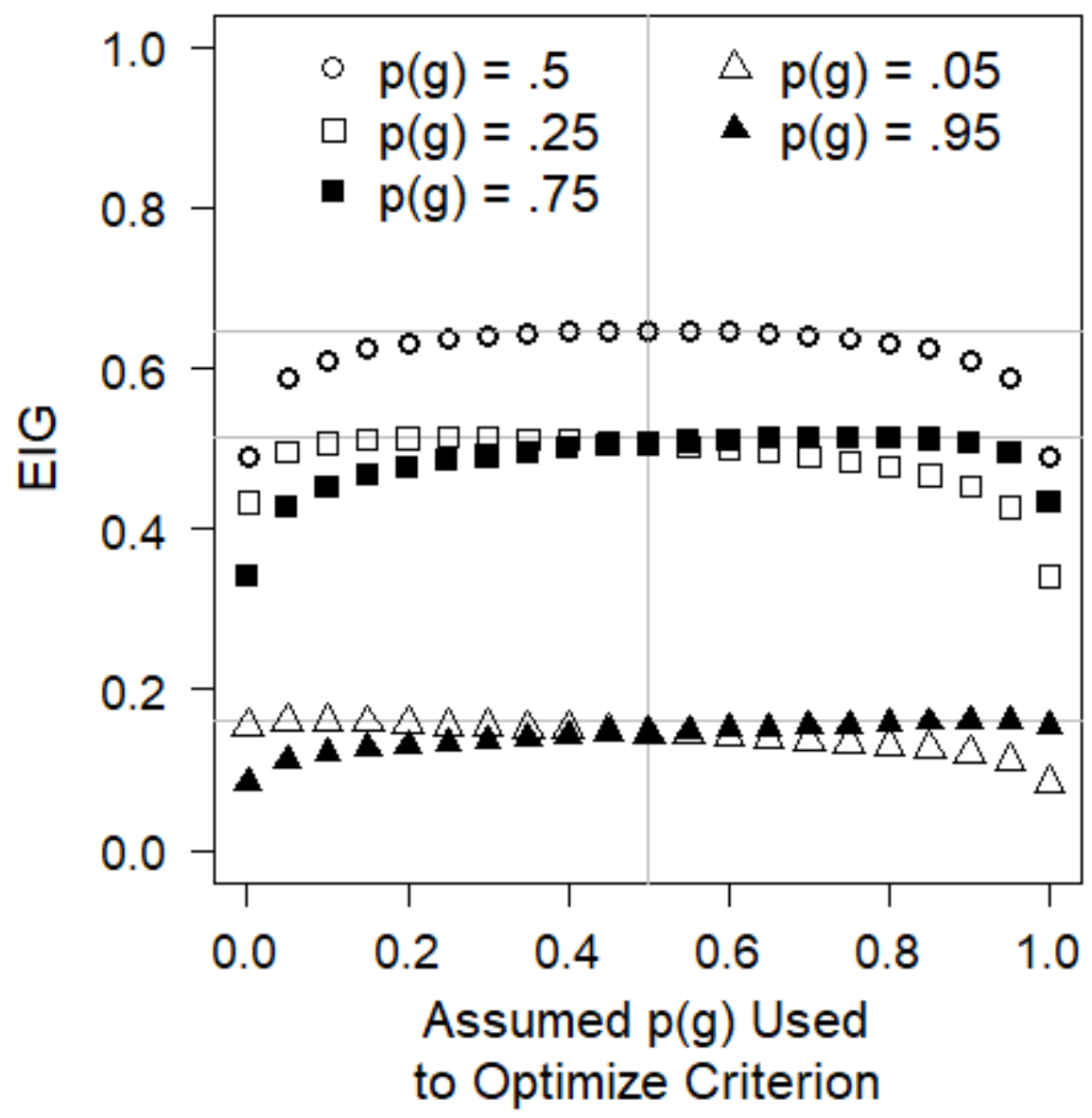

Figure 7.

EIG across different actual prior probabilities of guilt, $p(g)$, with criterion values optimized using different assumed prior probabilities of guilt. The model used Gaussian distributions with standard deviations of 1 and means of -1.5 and 1.5 for identification attempts with innocent and guilty suspects, respectively. Thus, $d^{\prime}$ was 3 . 


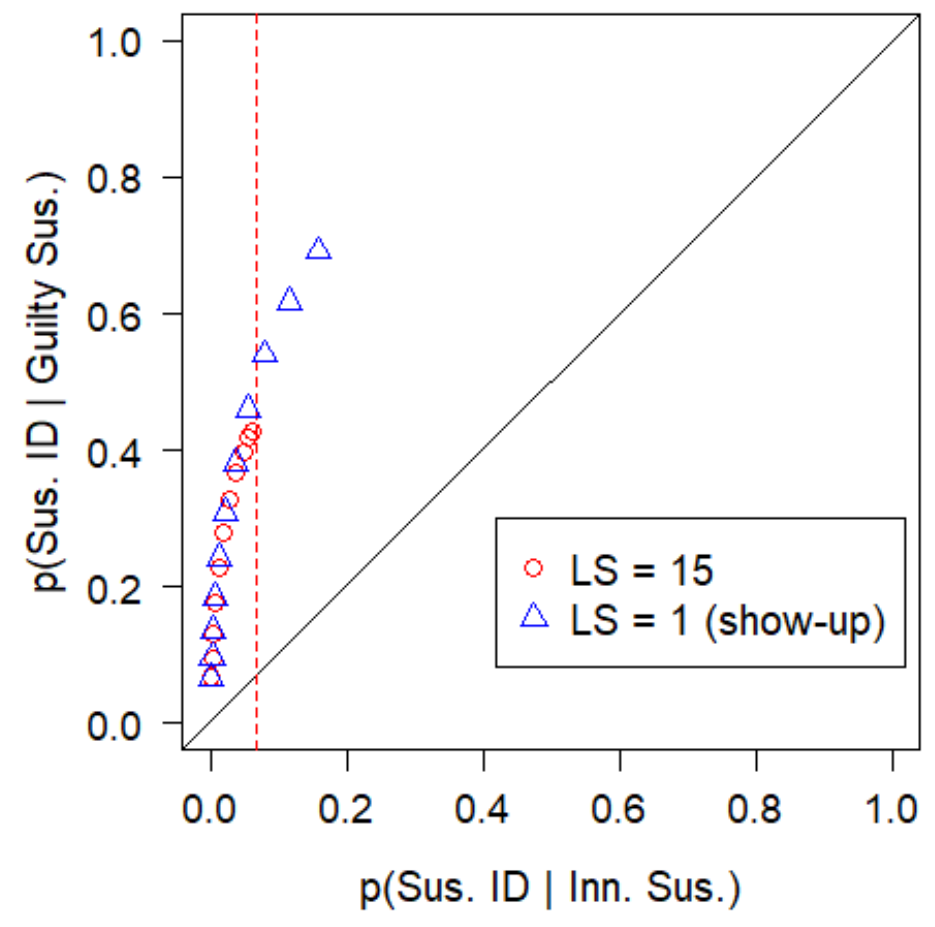

Figure 8.

ROCs for lineup sizes (LS) of 15 and 1. Identification decisions were based on a signal detection model in which the match-strength distribution had a mean of 1.5 for guilty suspects and 0 for innocent suspects (and fillers in the 15-person lineup). All distributions had a standard deviation of 1 . The identification criterion (minimum match to culprit required for an identification) was set to 1 , and 10 confidence criteria were arrayed above and below the identification criterion in equal steps of 0.20 to define confidence bins. For both identifications and rejections, the bin closest to the identification criterion was for confidence level 0 , the next bin out was for confidence level 10, and so forth until the most extreme bin for confidence level 100. Responses for the 15-person lineups were determined by selecting the lineup member with the highest match strength and comparing this member's strength values to the identification and confidence criteria. Sus. $=$ suspect $;$ ID $=$ identification; Inn. $=$ innocent. 


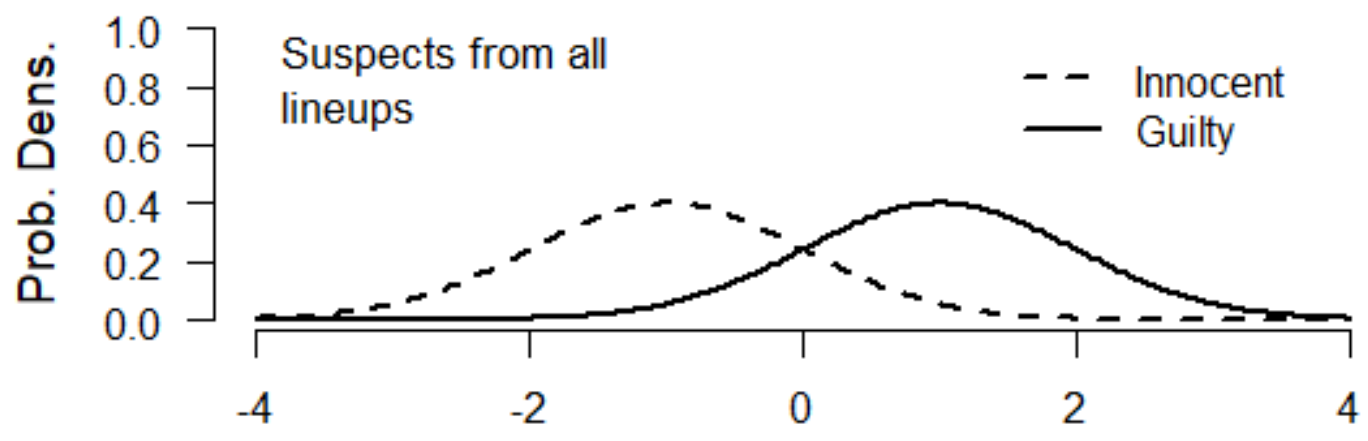

Match to Memory of Culprit

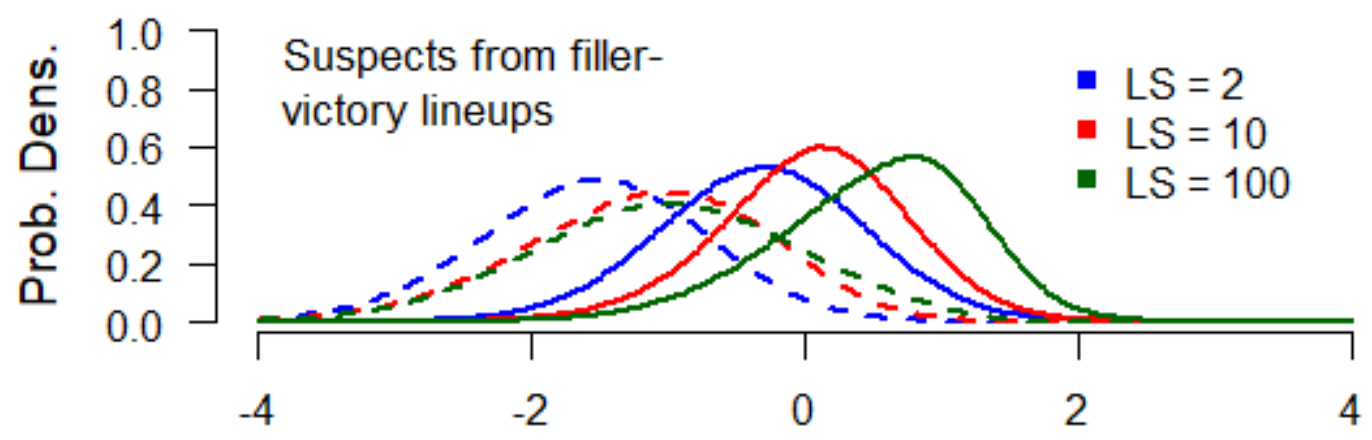

Match to Memory of Culprit

Figure 9.

Match strength distributions from a lineup signal-detection model across all suspects (top panel) or just suspects from lineups for which one of the fillers provided the strongest match to the witness' memory for the culprit (bottom panel). The model used Gaussian distributions with standard deviations of 1 and means of -1 and 1 for identification attempts with innocent and guilty suspects, respectively. The match strength distribution for fillers was identical to the distribution for innocent suspects. $L S=$ lineup size; Prob. Dens. = probability density. 

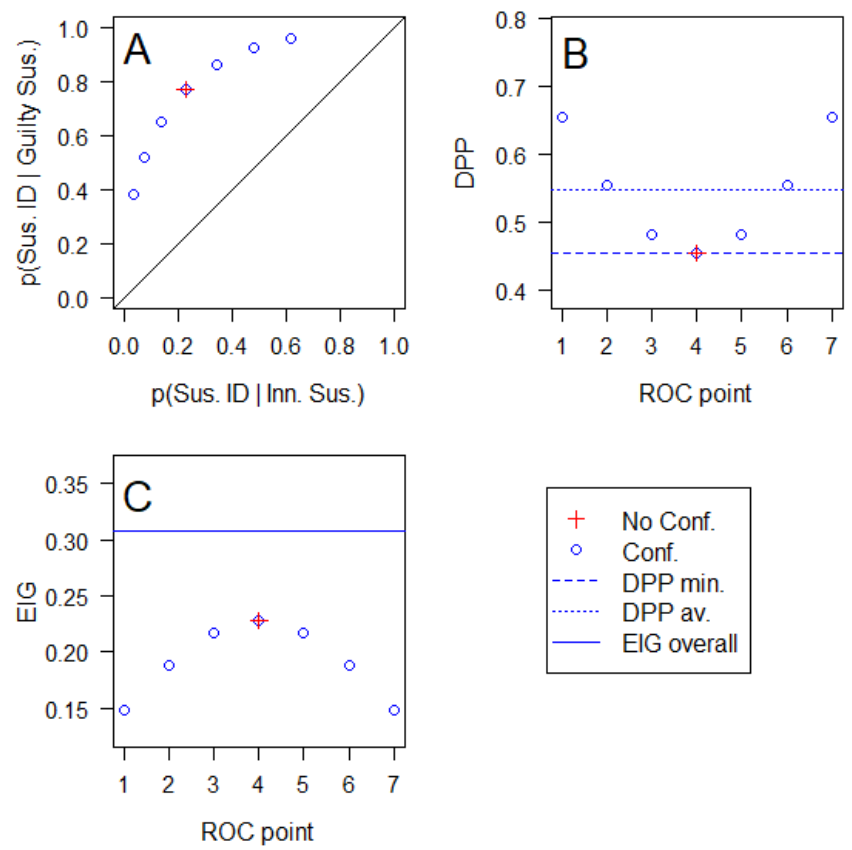

Figure 10.

ROC functions, deviation from perfect performance (DPP), and expected information gain (EIG) values. Identification decisions were based on a signal detection model in which the matchstrength distribution had a mean of 1.5 for guilty suspects and 0 for innocent suspects. Both distributions had a standard deviation of 1 . The identification criterion (minimum match to culprit required for an identification) was set to 0.75 , and 3 confidence criteria were arrayed above and below the identification criterion in equal steps of .35 to define confidence bins. For both identifications and rejections, the bin closest to the identification criterion was for confidence level 1 , the next bin out was for confidence level 2, and so forth until the most extreme bin for confidence level 4. Responses were determined by comparing the suspect's match strength to the identification and confidence criteria. Sus. = suspect; ID = identification; Inn. $=$ innocent $;$ min. $=$ minimum; av. $=$ average Conf. $=$ confidence . 

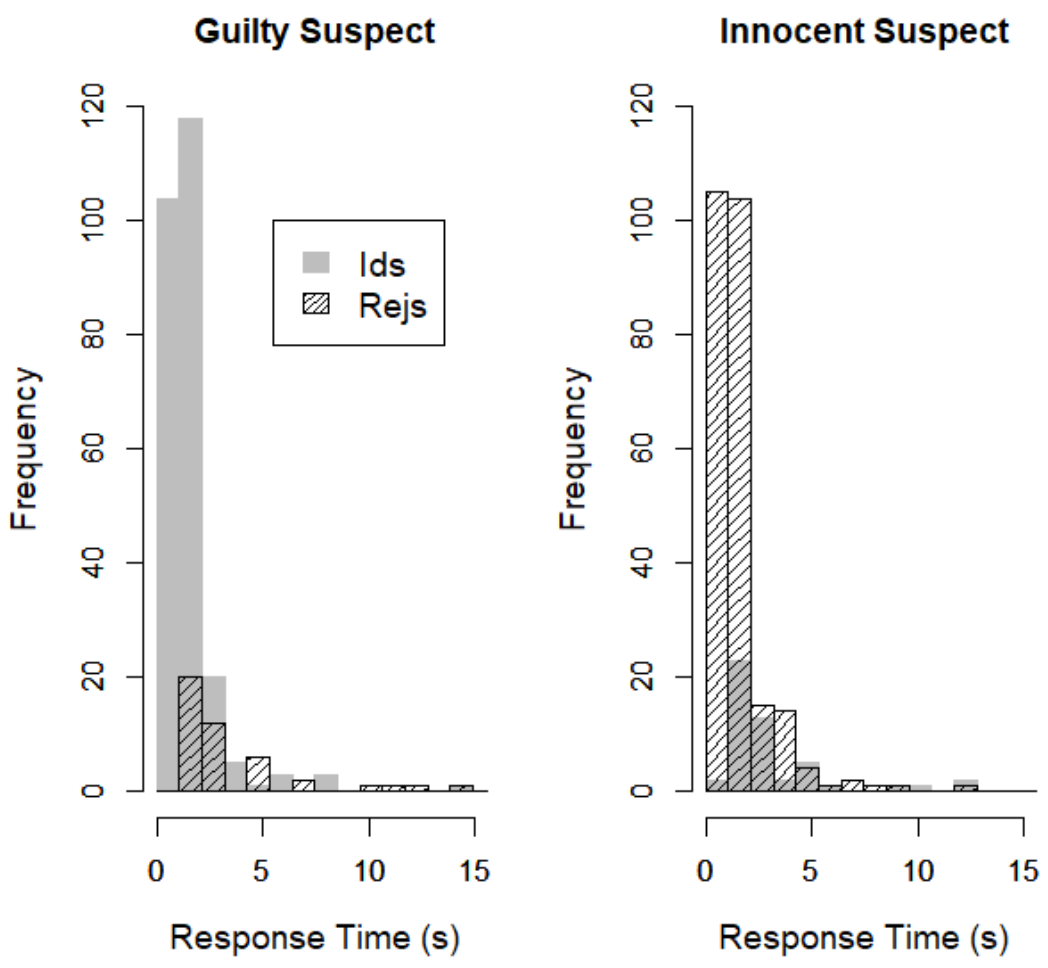

Figure 11.

Response time (RT) distributions for show-up data generated from the diffusion model. See Ratcliff \& McKoon (2008) for a detailed explanation of the model and the meaning of the parameters. The parameter values used to generate the data were $s$ (diffusion coefficient) $=1 ; a$ $($ boundary width $)=5 ; z($ relative starting point $)=.5 ; s z$ (variability in starting point $)=0 ; v_{G}$ (average drift rate for guilty-suspect trials $)=3 ; v_{I}$ (average drift rate for innocent-suspect trials) $=$ $-3 ; s v$ (variability in drift rate across trials $)=3 ; \operatorname{ter}($ mean non-decision time $)=.5 ;$ st (variability in non-decision time $)=.05$. Ids $=$ identifications; Rejs $=$ rejections . 


\section{Appendix}

Full table of EIG calculations for the 15-person lineup

\begin{tabular}{|c|c|c|c|c|c|c|c|}
\hline Response $(x)$ & $p(x \mid g)$ & $p(x \mid i)$ & $p(x)$ & $p(g \mid x)$ & $H(Y \mid x)$ & $I G(x)$ & $p(x) \times I G(x)$ \\
\hline Suspect Id. 100 & 0.066 & 0.001 & 0.0335 & 0.9851 & 0.1119 & 0.8881 & 0.0298 \\
\hline Suspect Id. 90 & 0.029 & 0.001 & 0.0150 & 0.9667 & 0.2108 & 0.7892 & 0.0118 \\
\hline Suspect Id. 80 & 0.037 & 0.002 & 0.0195 & 0.9487 & 0.2918 & 0.7082 & 0.0138 \\
\hline Suspect Id. 70 & 0.044 & 0.003 & 0.0235 & 0.9362 & 0.3425 & 0.6575 & 0.0155 \\
\hline Suspect Id. 60 & 0.050 & 0.005 & 0.0275 & 0.9091 & 0.4395 & 0.5605 & 0.0154 \\
\hline Suspect Id. 50 & 0.052 & 0.007 & 0.0295 & 0.8814 & 0.5255 & 0.4745 & 0.0140 \\
\hline Suspect Id. 40 & 0.049 & 0.009 & 0.0290 & 0.8448 & 0.6226 & 0.3774 & 0.0109 \\
\hline Suspect Id. 30 & 0.041 & 0.010 & 0.0255 & 0.8039 & 0.7140 & 0.2860 & 0.0073 \\
\hline Suspect Id. 20 & 0.030 & 0.010 & 0.0200 & 0.7500 & 0.8113 & 0.1887 & 0.0038 \\
\hline Suspect Id. 10 & 0.019 & 0.008 & 0.0135 & 0.7037 & 0.8767 & 0.1233 & 0.0017 \\
\hline Suspect Id. 0 & 0.010 & 0.006 & 0.0080 & 0.6250 & 0.9544 & 0.0456 & 0.0004 \\
\hline Filler Id. 100 & 0.018 & 0.019 & 0.0185 & 0.4865 & 0.9995 & 0.0005 & 0.00001 \\
\hline Filler Id. 90 & 0.015 & 0.016 & 0.0155 & 0.4839 & 0.9992 & 0.0008 & 0.00006 \\
\hline Filler Id. 80 & 0.025 & 0.028 & 0.0265 & 0.4717 & 0.9977 & 0.0023 & 0.0001 \\
\hline Filler Id. 70 & 0.038 & 0.045 & 0.0415 & 0.4578 & 0.9949 & 0.0051 & 0.0002 \\
\hline Filler Id. 60 & 0.054 & 0.068 & 0.0610 & 0.4426 & 0.9905 & 0.0095 & 0.0006 \\
\hline Filler Id. 50 & 0.071 & 0.096 & 0.0835 & 0.4251 & 0.9838 & 0.0162 & 0.0014 \\
\hline Filler Id. 40 & 0.082 & 0.122 & 0.1020 & 0.4020 & 0.9721 & 0.0279 & 0.0028 \\
\hline Filler Id. 30 & 0.084 & 0.138 & 0.1110 & 0.3784 & 0.9569 & 0.0431 & 0.0048 \\
\hline Filler Id. 20 & 0.073 & 0.137 & 0.1050 & 0.3476 & 0.9319 & 0.0681 & 0.0071 \\
\hline Filler Id. 10 & 0.054 & 0.115 & 0.0845 & 0.3195 & 0.9039 & 0.0961 & 0.0081 \\
\hline Filler Id. 0 & 0.032 & 0.079 & 0.0555 & 0.2883 & 0.8665 & 0.1335 & 0.0074 \\
\hline Rej. 100 & 0 & 0 & 0 & & . & - & --------- \\
\hline Rej. 90 & 0 & 0 & 0 & --------- & --------- & --------- & --------- \\
\hline Rej. 80 & 0 & 0 & 0 & --------- & ---- & & ---- \\
\hline Rej. 70 & 0 & 0 & 0 & --------- & ---- & --------- & --------- \\
\hline Rej. 60 & 0 & 0 & 0 & ---------- & ---------- & --------- & --------- \\
\hline Rej. 50 & 0 & 0 & 0 & ---------- & ---------- & ---------- & --------- \\
\hline Rej. 40 & 0 & 0 & 0 & --------- & ---------- & --------- & --------- \\
\hline Rej. 30 & 0 & 0.001 & 0.0005 & 0 & 0 & 1 & 0.0005 \\
\hline Rej. 20 & 0.002 & 0.006 & 0.004 & 0.25 & 0.8113 & 0.1887 & 0.0008 \\
\hline Rej. 10 & 0.007 & 0.020 & 0.0135 & 0.2593 & 0.8256 & 0.1744 & 0.0024 \\
\hline Rej. 0 & 0.019 & 0.047 & 0.033 & 0.2879 & 0.8660 & 0.1340 & 0.0044 \\
\hline
\end{tabular}

$\Sigma p(x) \times I G(x)=E I G=0.16$

Notes. Model predictions were rounded to the nearest $1000^{\text {th }}$, resulting in zero probability for some responses. Zero-probability responses are ignored in the $E I G$ calculations. $p(x \mid g)=$ 
probability of Response $x$ on identification attempts with guilty suspects; $p(x \mid i)=$ probability of Response $x$ on identification attempts with innocent suspects; $p(x)=$ overall probability of Response $x ; p(g \mid x)=$ posterior probability of guilt for Response $x$ (posterior probability of innocence is $1-p(g \mid x)) ; H(Y \mid x)=$ entropy for Response $x ; I G(x)$ information gain for Response $x$; $\mathrm{EIG}=$ expected information gain. 